\title{
A Data-Input Program (MFI2005) for the U.S. Geological Survey Modular Groundwater Model (MODFLOW-2005) and Parameter Estimation Program (UCODE_2005)
}

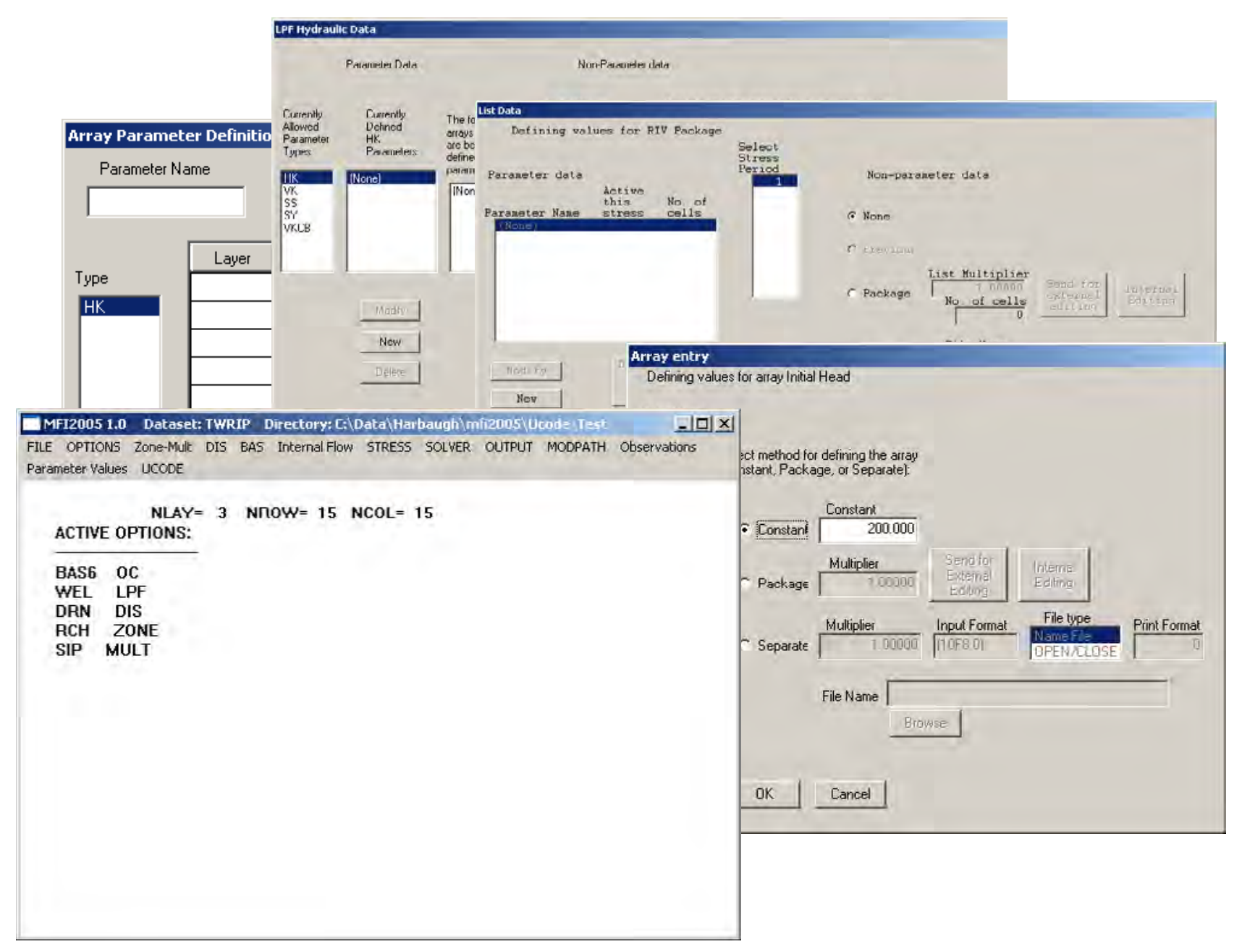

Open-File Report 2010-1057

U.S. Department of the Interior

U.S. Geological Survey 



\section{A Data-Input Program (MFI2005) for the U.S. Geological Survey Modular Groundwater Model (MODFLOW-2005) and Parameter Estimation Program (UCODE_2005)}

By Arlen W. Harbaugh

Open-File Report 2010-1057 


\title{
U.S. Department of the Interior \\ KEN SALAZAR, Secretary \\ U.S. Geological Survey \\ Marcia K. McNutt, Director
}

\section{U.S. Geological Survey, Reston, Virginia: 2010}

\author{
For more information on the USGS — the Federal source for science about the Earth, its natural and living resources, \\ natural hazards, and the environment, visit http://www.usgs.gov or call 1-888-ASK-USGS \\ For an overview of USGS information products, including maps, imagery, and publications, \\ visit http://www.usgs.gov/pubprod \\ To order this and other USGS information products, visit http://store.usgs.gov
}

Any use of trade, product, or firm names is for descriptive purposes only and does not imply endorsement by the U.S. Government.

Although this report is in the public domain, permission must be secured from the individual copyright owners to reproduce any copyrighted materials contained within this report.

Suggested citation:

Harbaugh, A.W., 2010, A data-input program (MFI2005) for the U.S. Geological Survey modular groundwater model (MODFLOW-2005) and parameter estimation program (UCODE_2005): U.S. Geological Survey Open-File Report 2010-1057, 35 p. 


\title{
Preface
}

This report describes a computer program called MFI2005 that can be used to prepare input data for the U.S. Geological Survey groundwater model (MODFLOW-2005). The code is in the public domain and can be downloaded from the Internet at http://water.usgs.gov/software/ground water.html. The code has been tested in a variety of applications, but future applications could reveal previously undetected errors. Users are requested to notify the originating office of any errors found in the report or computer code:

\author{
U.S. Geological Survey \\ Office of Ground Water \\ 411 National Center \\ Reston, VA 20192 \\ (703) 648-5001
}

Updates to the code will be posted at the above Internet address. 


\section{Contents}

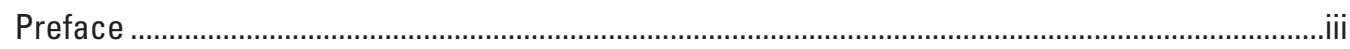

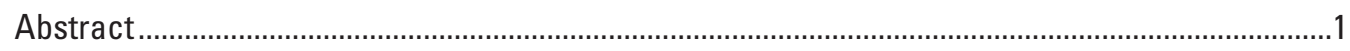

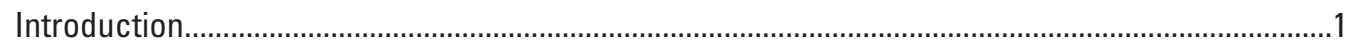

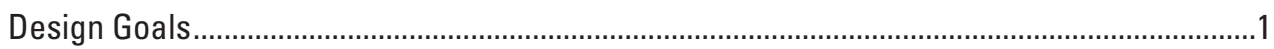

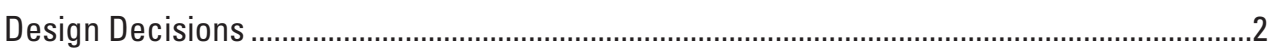

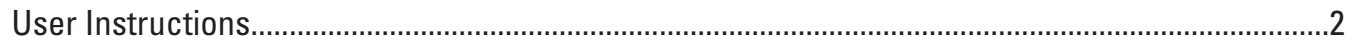

MODFLOW-2005 Capabilities Supported by MFI2005 .........................................................

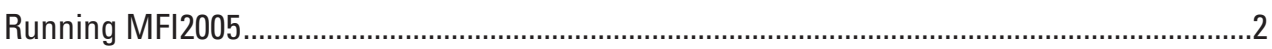

Main Menu

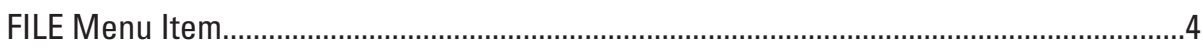

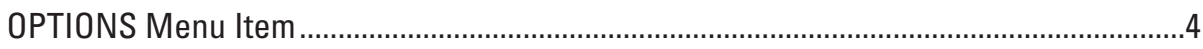

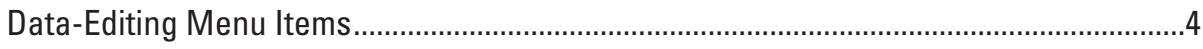

Entering Two-Dimensional Arrays ...............................................................................4

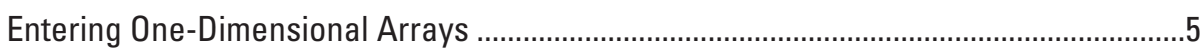

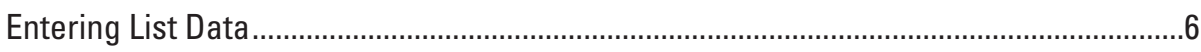

Entering Parameters ….............................................................................................

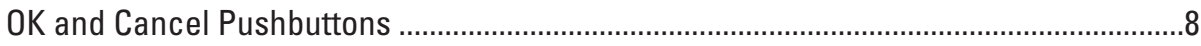

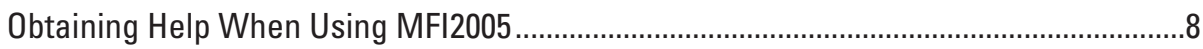

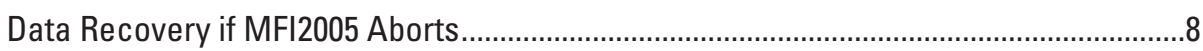

Entering Data with an External Editing Program .........................................................

Running MODFLOW-2005, MODPATH, and UCODE_2005 ......................................................

Miscellaneous Details About Using MFI2005 ……………….................................................

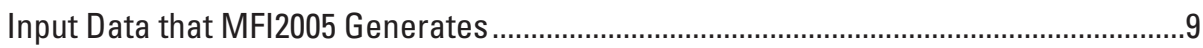

Input Data Shared Between MODFLOW and MODPATH.................................................

Constraints in MFI2005 That Are Not in MODFLOW-2005 and MODPATH .....................10

Using MFI2005 When MODFLOW-2005 Includes Capabilities

That MFI2005 Does Not Include.......................................................................10

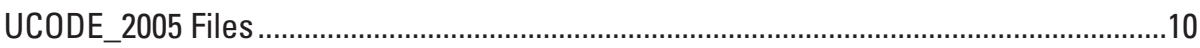

Using MFI2005 With a Dataset Not Originally Created by MFI2005 .................................11

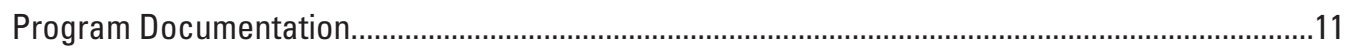

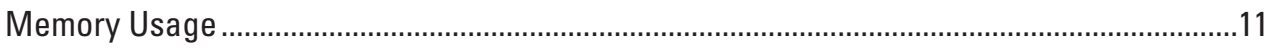

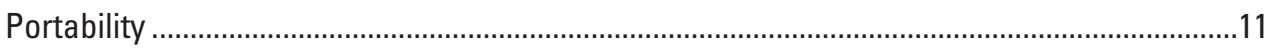

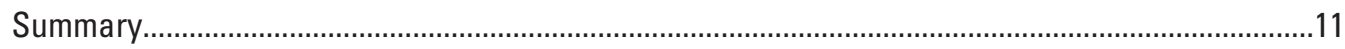

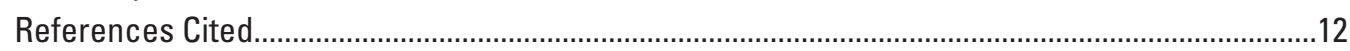

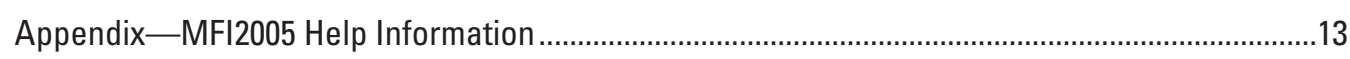

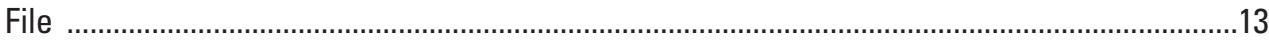

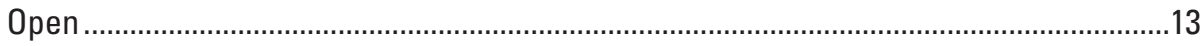

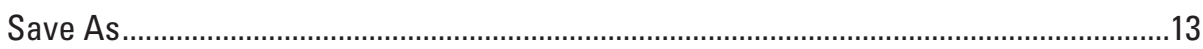

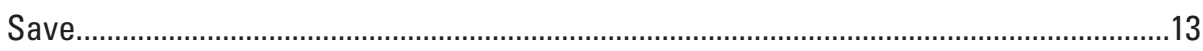

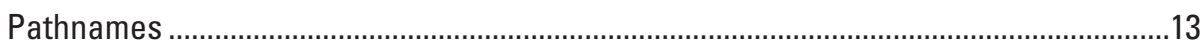

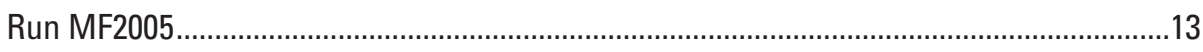

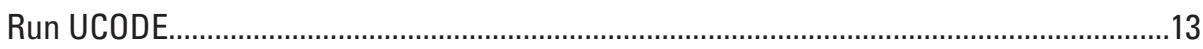

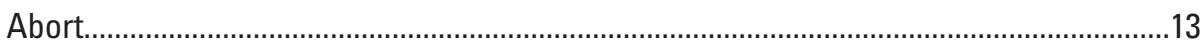

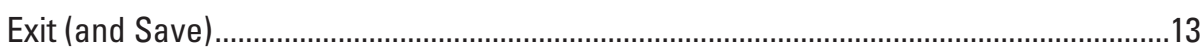




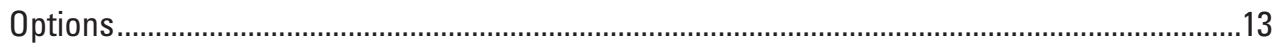

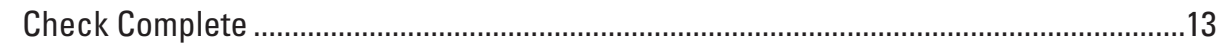

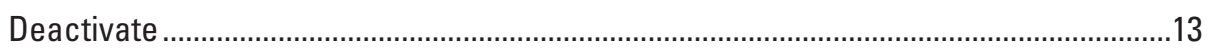

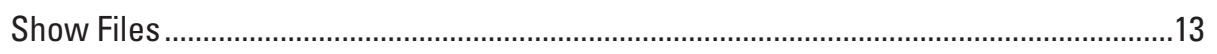

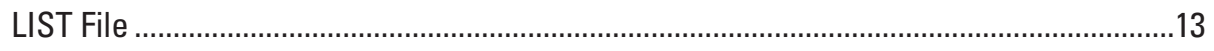

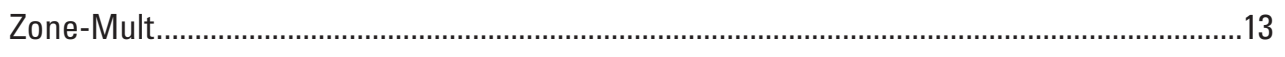

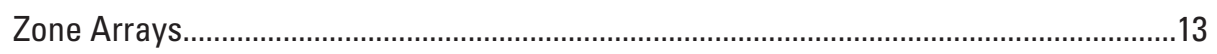

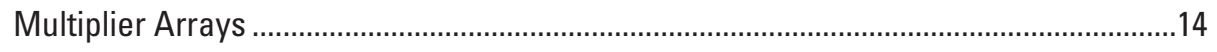

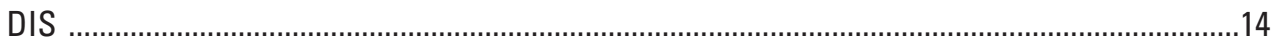

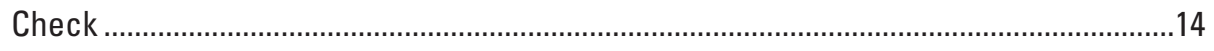

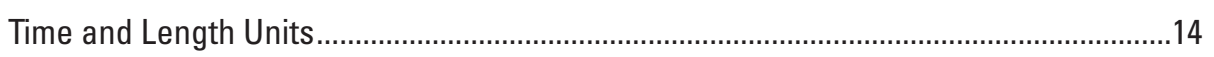

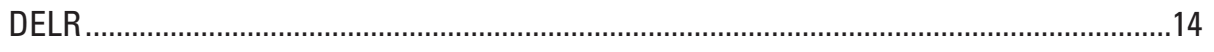

DELC

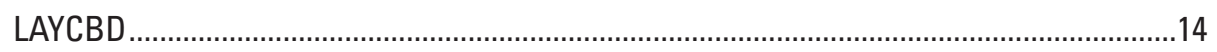

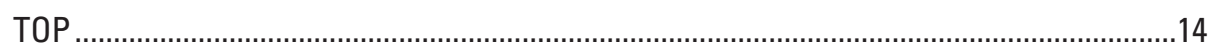

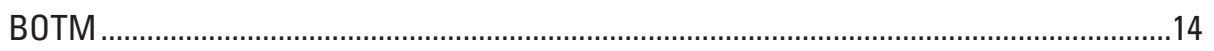

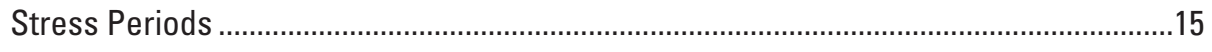

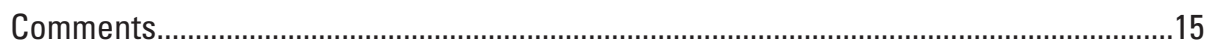

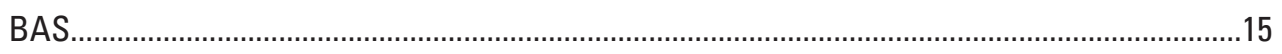

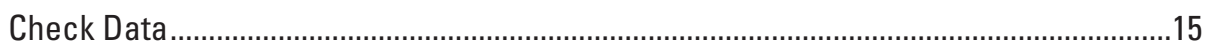

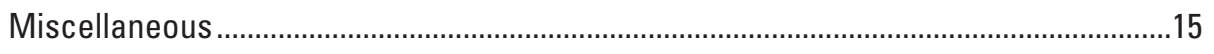

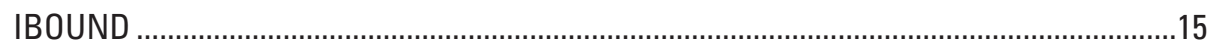

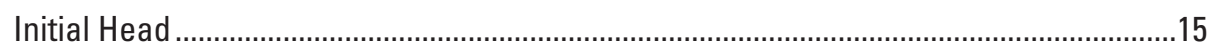

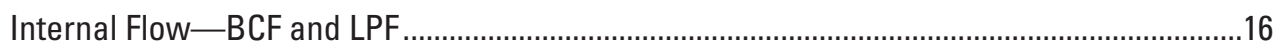

BCF-Block-Centered Flow Package .....................................................................16

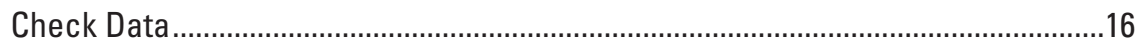

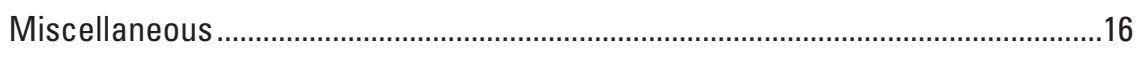

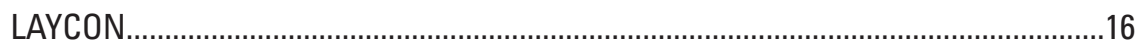

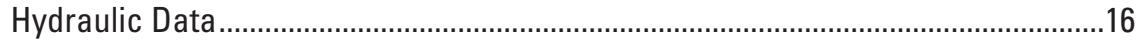

LPF_Layer-Property Flow Package ........................................................................17

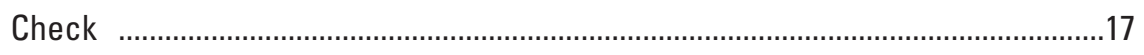

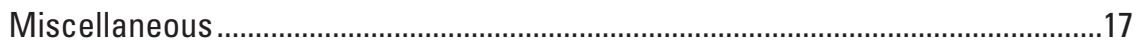

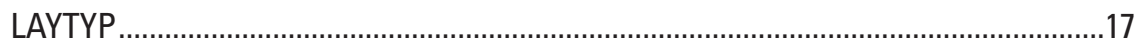

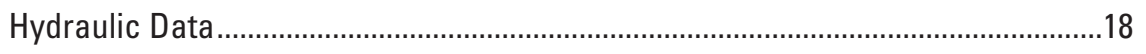

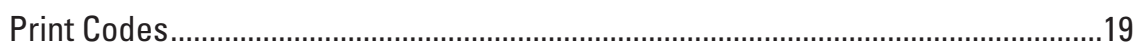

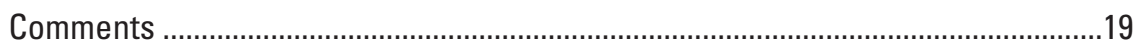

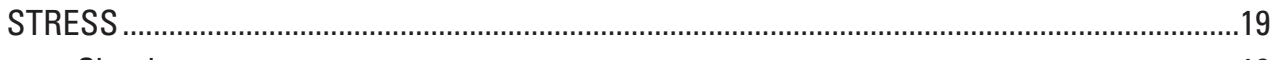

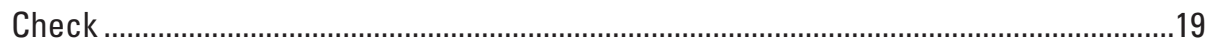

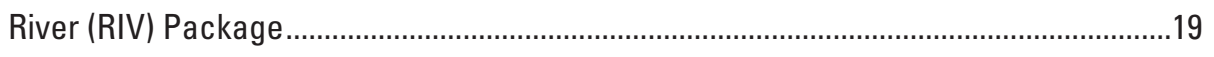

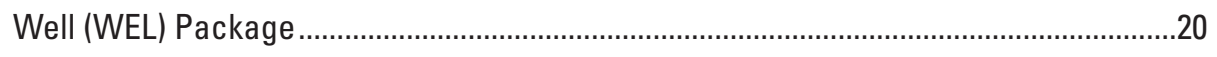

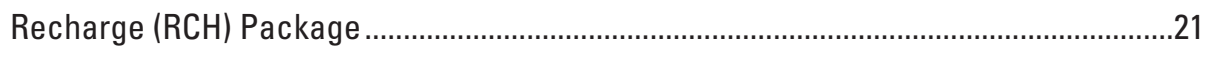

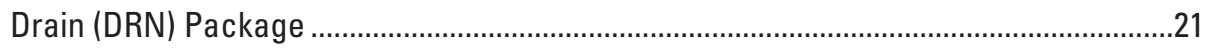

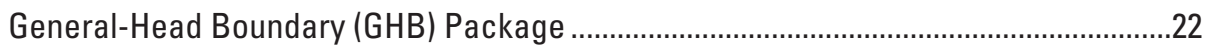

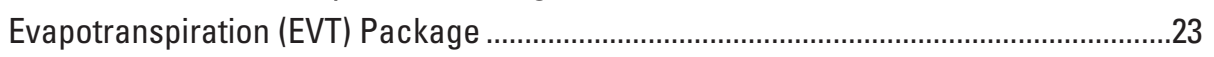




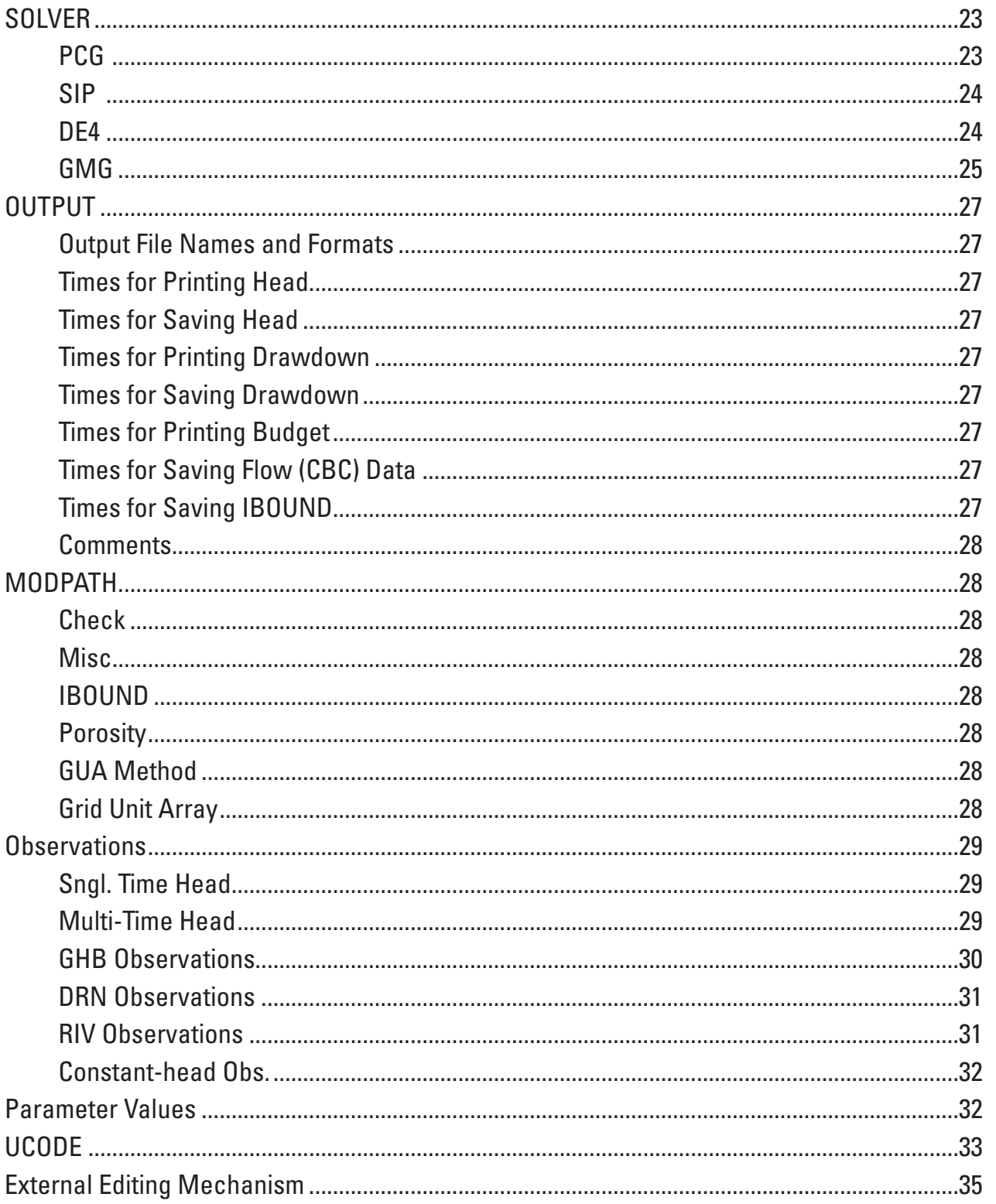

\section{Figures}

1-5. Screen captures showing-

1. Main Menu for data-input program MFI2005 ….......................................................

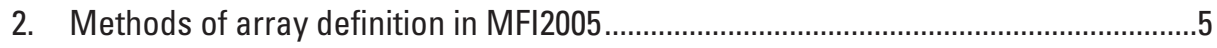

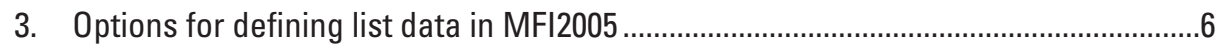

4. Array definition in MFI2005 .........................................................................................

5. Definition of array parameters in MFI2005.............................................................. 



\title{
A Data-Input Program (MFI2005) for the U.S. Geological Survey Modular Groundwater Model (MODFLOW-2005) and Parameter Estimation Program (UCODE_2005)
}

\author{
By Arlen W. Harbaugh
}

\begin{abstract}
The MFI2005 data-input (entry) program was developed for use with the U.S. Geological Survey modular three-dimensional finite-difference groundwater model, MODFLOW-2005. MFI2005 runs on personal computers and is designed to be easy to use; data are entered interactively through a series of display screens. MFI2005 supports parameter estimation using the UCODE_2005 program for parameter estimation. Data for MODPATH, a particle-tracking program for use with MODFLOW-2005, also can be entered using MFI2005. MFI2005 can be used in conjunction with other data-input programs so that the different parts of a model dataset can be entered by using the most suitable program.
\end{abstract}

\section{Introduction}

Computer models of groundwater flow require input data in order to define the simulated flow system. Most modeling programs input these data from one or more files rather than prompting for the data interactively. The reason for this method is partly evolutionary. When groundwater models were first developed in the 1960s, the primary focus was on developing the numerical methods to solve the differential equations of flow. The major challenge was being able to solve practical, real-world problems within the constraints of computer speed and memory. The simulations generally were small by present (2010) standards; therefore, preparing the data files by using a card punch or text editor was easy. Further, computers in the 1960s were not designed to allow for interactive input.

Presently, computers easily can handle large simulation problems where data preparation is a burdensome task. Considerable benefit can be obtained from an interactive program that helps prepare model-input data. The interactive program can prompt for the required data, check for errors, and ensure that files are created with the proper structure. Although adding interactive data entry to the modeling program is possible, it is not desirable because of the required increased memory and program complexity. Furthermore, the wide variations in user preferences and needs make it unlikely that any single method of interactive data input will meet all needs. Having data entry separate from the model allows different data-entry programs to be developed independently of the modeling program.

This report describes MFI2005, a data-input program for MODFLOW-2005, which is the U.S. Geological Survey (USGS) three-dimensional finite-difference groundwater model (Harbaugh, 2005). In addition, MFI2005 can prepare data for UCODE_2005, which is a parameter-estimation program (Poeter and others, 2005), and MODPATH, which is a particle-tracking program that uses flow calculated by MODFLOW-2005 (Pollock, 1994). MFI2005 is based on the MFI2K program (Harbaugh, 2002), which is a data-input program for the MODFLOW-2000 (Harbaugh and others, 2000). Instructions for using MFI2005 are also included.

Users of MFI2005 are assumed to be familiar with the design and input requirements of MODFLOW-2005. Users also are assumed to understand basic groundwater modeling concepts.

\section{Design Goals}

The primary design goals for MFI2005 are as follows:

1. Easy to use-Although this goal is subjective, some of the desired attributes are as follows: an intuitive user interface; the user is guided in a logical manner through the data entry process; and checking is done to make sure valid or reasonable data are entered.

2. Compatible with other data-entry programs-Modelers use a variety of computer applications to help manipulate data. For example in the USGS, many modelers use geographic information system (GIS) software to store and manipulate data. It is important to be able to use data from the GIS along with data entered by using MFI2005.

3. Low development costs.

4. Useful for a wide range of modeling problems from simple training exercises to large modeling projects.

5. Runs on personal computers. 


\section{Design Decisions}

The following design decisions were made in order to fulfill the design goals:

1. Fortran is the programming language - Many other languages would have been adequate for programming, but the author is most familiar with Fortran. Also, MODFLOW-2005 is written in Fortran; therefore, reading and writing data for MODFLOW is easy.

2. The Winteracter Subroutine Library (Interactive Software Services, 2003) is used as the tool for providing user interaction in MFI2005. Winteracter provides a set of tools for creating Fortran programs that interact with users through Microsoft Windows ${ }^{\circledR}$.

3. Spatial data will be entered into MFI2005 by using grid coordinates rather than arbitrary geographic coordinates that require transformation in order to determine grid coordinates. Also, data values for the grid must be entered into MFI2005 for all locations required by MODFLOW-2005 rather than entering fewer values and having MFI2005 interpolate the missing values. Transformation to grid coordinates and data interpolation capabilities can be obtained by using other programs in addition to MFI2005.

4. As a further method of allowing the user to maximize the use of other software, MFI2005 will allow external files to be specified as part of a MODFLOW-2005 dataset. These external files will not be read or modified by MFI2005, but the dataset will be set up so that MODFLOW-2005 will read these files. The use of external files in MFI2005 is possible because MODFLOW-2005 allows arrays to be read from external files.

5. To avoid duplicate data, MFI2005 will store all MODFLOW-2005 data in the MODFLOW-2005 input files rather than in a separate database. This will allow MFI2005 to edit MODFLOW-2005 data that were not initially prepared using MFI2005. MFI2005 is unable to read data from UCODE_2005 input files, so MFI2005 cannot edit UCODE_2005 data prepared outside of MFI2005. UCODE_2005 data entered through MFI2005, however, are saved in a separate MFI2005 file so that MFI2005 can obtain the data when MFI2005 is run subsequently.

\section{User Instructions}

The MFI2005 program displays screens to which the user responds. The user enters data, makes a choice, or reads displayed information. These user interactions are conducted by using the keyboard and mouse as is typical in interactive programs on personal computers.

\section{MODFLOW-2005 Capabilities Supported by MFI2005}

The capabilities of MODFLOW-2005 that are supported by the MFI2005 program are as follows:

- Discretization (DIS) File

- Basic (BAS) Package

- Block-Centered Flow (BCF) Package

- Layer-Property Flow (LPF) Package

- Well (WEL) Package

- Drain (DRN) Package

- River (RIV) Package

- Evapotranspiration (EVT) Package

- General-Head Boundary (GHB) Package

- Recharge (RCH) Package

- Strongly Implicit Procedure (SIP) Package

- Preconditioned Conjugate Gradient (PCG) Package (Hill, 1990)

- Direct Solution (DE4) Package (Harbaugh, 1995)

- Geometric Multigrid (GMG) Package (Wilson and Naff, 2004)

- Output Control (OC) Option

- Parameter Value File

- Observation Process

- UCODE_2005

\section{Running MFI2005}

MFI2005 resides on a personal computer as executable file MFI2005.exe. This file can be executed by using a Microsoft Windows ${ }^{\circledR}$ shortcut, using Windows Explorer ${ }^{\circledR}$, or entering the following command in a command window:

MFI2005

or

MFI2005 NameFile, where NameFile is the MODFLOW Name File of a new or existing dataset.

If the name file is not entered as part of the command, then the first screen to appear will prompt for this. MFI2005 only accepts name files that end with ".nam"; however, the ".nam" part of the name need not be entered because MFI2005 will automatically add it, if necessary. The part of the file name before ".nam" is called the dataset name and can be from 1 to 28 characters.

The dataset name is used as the prefix for many file names that MFI2005 creates. These file names are constructed 
by adding a period plus two or three characters to the dataset name. Some examples are

- $\log$ is the suffix for a $\log$ file of information generated by MFI2005,

- ba6 is the suffix for the Basic Package file of MODFLOW-2005,

- .bc6 is the suffix for the BCF Package file of MODFLOW-2005,

- .mpn is the suffix for the MODPATH name file,

- .mp is the suffix for the main MODPATH data file, and

- .mfi is the suffix for the file in which MFI2005 saves data used to generate UCODE_2005 files.

The dataset name cannot include characters that would be invalid as part of a file name on the particular computer and operating system being used.

If a name file exists, it is read along with all of the MODFLOW input data, and the Main Menu is displayed. If a name file is new, the next screen prompts for confirmation that it is acceptable to create a new model dataset. If it is not acceptable to create a new dataset, MFI2005 stops without performing other tasks. MFI2005 stops at this point because it is assumed that the user intended to enter the name of an existing dataset. The user then can restart MFI2005. If
MFI2005 is permitted to create a new dataset, the next screen prompts for the number of layers, rows, and columns. These grid size values cannot be changed within MFI2005 after this screen is accepted; therefore, care should be given to specify them properly. This screen also allows the choice to be made between free and fixed format, which refers to the structure of MODFLOW data files. Free format means that the width of each data field can vary, whereas fixed format means that the field width for each value is fixed. This option applies only to those packages that support both formats.

\section{Main Menu}

Once the dataset name is established, a window is created that includes the Main Menu at the top and information about the active simulation options (fig. 1). Most of the menu items apply to data entry; however, "FILE" provides typical functions for saving data and exiting the program, and "OPTIONS" provides useful information, such as file names of all files that will be used in the MODFLOW-2005 simulation.

The data-editing menu items generally are organized according to MODFLOW files. For example, "Zone-Mult" allows entry of data in the files that specify zone and multiplier arrays. "DIS" allows entry of data for the discretization file. To limit the number of choices in the Main Menu, many of the menu items have submenus. For example, "Internal

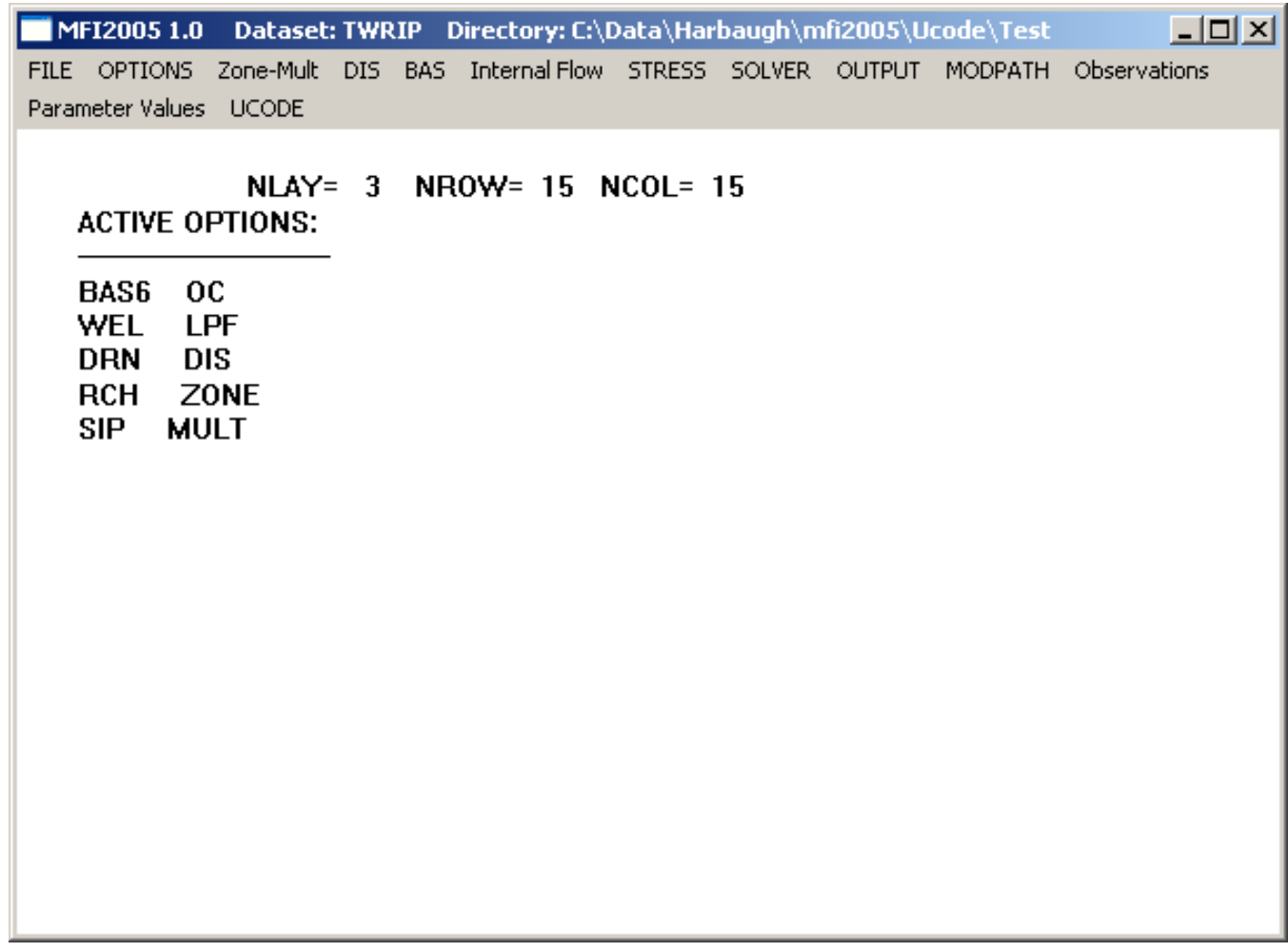

Figure 1. Main Menu for data-input program MFI2005. 
Flow" contains items that allow entry of data for the BlockCentered Flow (BCF) and Layer-Property Flow (LPF) Package files. The stress packages are combined into a single menu item, "STRESS." The solvers for which MFI2005 can prepare data are included in the single "SOLVER" item. "MODPATH" also is a menu item, which allows entry of data needed for the MODPATH Main Data file.

\section{FILE Menu Item}

Selecting the "FILE" menu item causes a submenu to be displayed with the following items: "Open," "Save As," "Save," "Pathnames," "Run MF2005," "Run UCODE," "Abort," and "Exit (and Save)." "Open" allows a new dataset to be opened. The "Save As" item saves the dataset using a new dataset name, including the name file. All files, including the listing file, package files, option files, and process files, are renamed. The "Save" item saves the dataset using the current name. The "Pathnames" item allows the pathnames for running MODFLOW-2005 and UCODE_2005 to be specified. Default names are MF2005 and UCODE_2005. The "Run MF2005" item saves data using the current name and runs MODFLOW-2005. The "Run UCODE" item saves data using the current name and runs UCODE_2005. The "Abort" item exits MFI2005 without saving data. The "Exit (and Save)" item saves data and then exits the program.

\section{OPTIONS Menu Item}

Selecting the "OPTIONS" menu item causes a submenu to be displayed with the following items: "Check Complete," "Deactivate," "Show Files," and "LIST File." The "Check Complete" item indicates whether or not all of the data are defined for each active option. If an option is designated as "incomplete," then some of the data have not been specified. The specific undefined data can be displayed for an option by selecting the appropriate editing item from the Main Menu. For example, if Block-Centered Flow (BCF) data are incomplete, select BCF from the "Internal Flow" submenu. This selection will cause a BCF dialog to appear that includes a "Check Data" option. Selecting that option will display all undefined data for the BCF Package. The user may be surprised that some data that have not been entered do not show as undefined. This occurs because MFI2005 automatically specifies defaults for many values. These values can be changed if desired, but they need not be defined explicitly by the user if the defaults are adequate. The "Deactivate" item allows simulation options to be turned off. The "Show Files" item displays all files that have been defined and indicates their use. The "LIST File" item allows the name of the Listing File for the simulation to be specified.

The "Deactivate" item is provided because the dataediting menus do not provide a mechanism for turning options off. Once data entry is initiated for any MODFLOW option, MFI2005 will activate that option and write data files for that option when data are saved. When MODFLOW runs, that option will be invoked. Likewise, if data entry for MODPATH is initiated, MODPATH data files will be written. If an option is deactivated, the data for that option will no longer be saved when MFI2005 saves data. Also, MODFLOW and MODPATH will not include that option when they are executed. However, if a file for a deactivated option was present prior to its deactivation, that file will still be present after MFI2005 exits.

\section{Data-Editing Menu Items}

Selecting a data-editing menu item invokes a corresponding dialog screen called a primary dialog. Most primary dialogs consist of a list of options that activate other dialog screens for the actual data entry. The primary dialogs that can activate additional screens have a CLOSE option, which will return the user to the Main Menu. The user must ultimately return to the Main Menu in order to exit MFI2005. Some of the primary dialogs include a "Check" item, which displays information about undefined data.

\section{Entering Two-Dimensional Arrays}

Three methods can be used to define two-dimensional arrays in MFI2005. These methods correspond to the three methods that MODFLOW and MODPATH use for defining arrays. The first method, referred to as the Constant Method, is to make the entire array a constant.

The second method, called the Package Method, is to define individual values for each array element that MFI2005 writes into the appropriate MODFLOW file. This file typically is a package file, hence the name Package Method; however, data also are written into other files as appropriate such as the file for the multiplier arrays that are used to define parameters. When the Package Method is used, the user enters the values for the individual array elements by using an internal array editing capability or an external data-editing program. For Internal Editing, a screen appears with the current array values. The user can change these values as desired. The Internal Editing screen is limited to 100 rows and 100 columns. If there are more than 100 rows or columns in the grid, the Internal Editing option cannot be selected. The Internal Editing functionality is not as versatile as the functionality found in many spreadsheet programs. Accordingly, Internal Editing is adequate for entering small arrays and for quick examination and minor changes of large arrays; however, External Editing is likely more useful for initial entry and major modifications of large arrays. The mechanism for External Editing is described below in the Entering Data with an External Editing Program section.

The third method, called the Separate Method, is to specify that the values for individual array elements are in a separate file that MFI2005 does not read or write. Although MFI2005 does not actually read or write the contents of such files, MFI2005 incorporates information about these files into 
the proper locations in the dataset so that MODFLOW and MODPATH will read them. The files must be prepared using other software.

All three methods of defining arrays are specified on the same screen. An example screen that defines an array is shown in figure 2. In this example, the Constant Method is being used. The other two methods can be selected by clicking on the appropriate option (called a radio button). Notice that for each method there are different data fields that can be specified. The fields for a method cannot be modified unless the method first is selected. For the Package and Separate Methods, a constant is specified that is multiplied with each element of the array to make the final value. Thus, the entire array can be rescaled by changing the multiplier. Note that the array multiplier is different from a multiplier array, which is used for defining parameters.

For arrays defined using the Separate Method, the information that will allow MODFLOW or MODPATH to read the array must be specified. This information includes the file name, an input format, and a code to indicate which format to use when printing the array after it is read. Remember that MFI2005 does not read values for these arrays; therefore, if errors occur, such as an incorrect format or an invalid file name, they will not be detected by MFI2005. Such errors will not be detected until MODFLOW or MODPATH is executed. Choose a file type, which can be either "OPEN/ CLOSE" or "Name File." This choice corresponds to the capability of MODFLOW to declare the name of a data file in either the array control record or the MODFLOW name file
(Harbaugh, 2005, p. 8-57). If a file is used to define a single array, either file type can be used. If a file is used for more than one array, the file type must be the same for all the arrays, and it is important to specify the proper file type. If the identical data are to be specified for all of the arrays defined by the file, the file type should be "OPEN/CLOSE." For this file type, the same file will be reopened each time it is used to define an array, which causes the same data to be read over and over. If the same file contains multiple arrays, each of which may have different values, the file type must be "Name File." This type of file is left open as each array is read so that the multiple arrays are read in sequence from the file.

\section{Entering One-Dimensional Arrays}

One-dimensional arrays are defined in the same way as two-dimensional arrays. That is, the same three methods, Constant, Package, and Separate, can be selected. Although the amount of data to be entered is less than for a two-dimensional array, the input methods are the same. When using the Package Method, the data can be edited from an internal editing screen or an external editing program. The data will display as either a row or column depending on the type of data. For example, the grid spacing along a row (DELR) displays as one row, with one value for each column in the model grid. Similarly, column (DELC) values display as one column with one value for each row in the model grid.

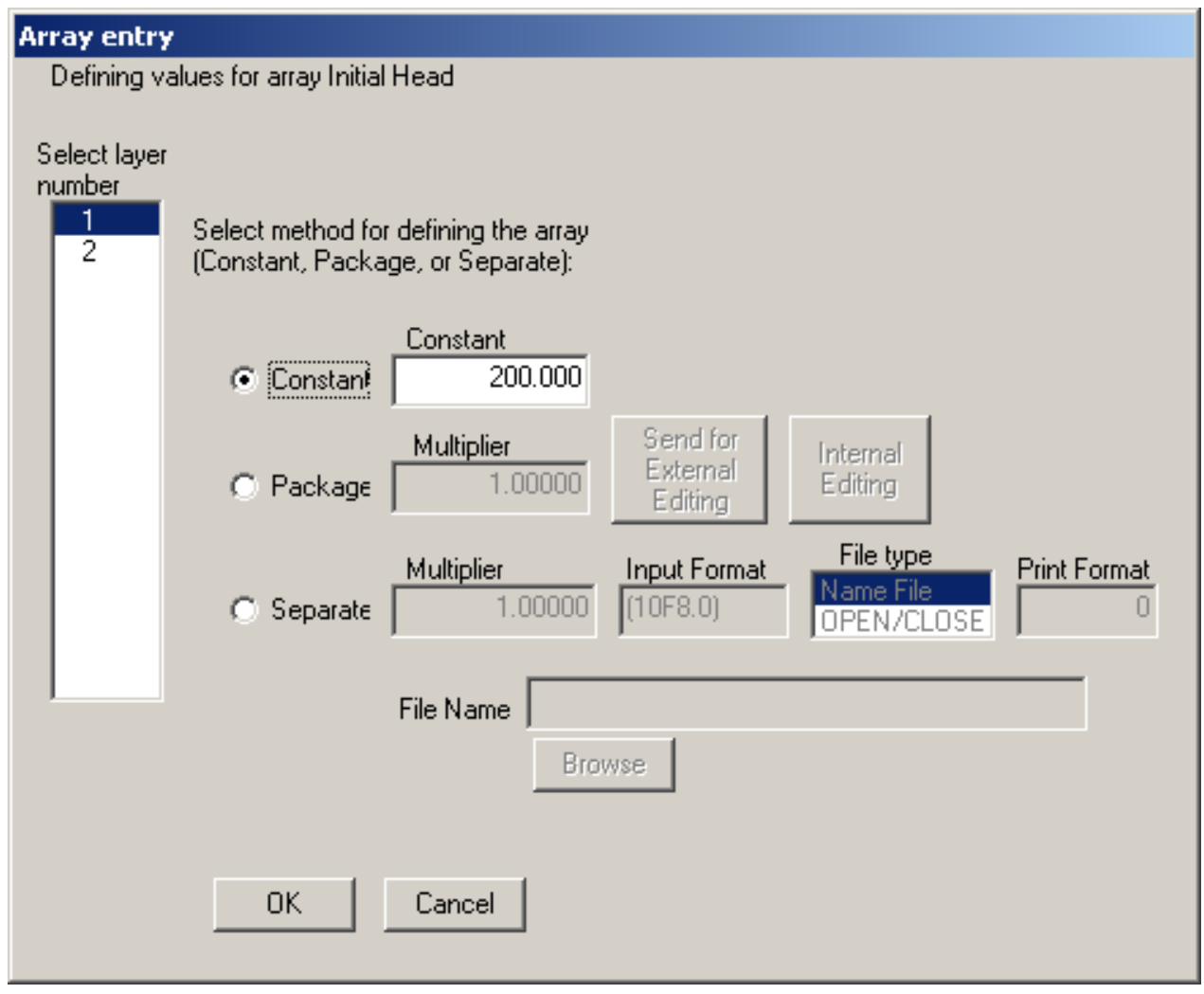

Figure 2. Example screen showing methods of array definition in MFI2005. 


\section{Entering List Data}

"List data" refers to data that are specified in a MODFLOW file for a group of cells using one line of data for each cell. A line of data includes the layer, row, and column indices for one cell plus some additional information. For example, list data are used to define wells and river reaches. In MFI2005, four methods can be used to define list data: None, Previous, Package, and Separate. The None Method indicates that the list has no entries. The Previous Method indicates that the list from the previous stress period should be reused. This method is not available for the first stress period. The Package and Separate Methods are similar to the methods of the same names for arrays. The Package Method allows the list of data to be edited by using an internal editing screen or an external editing program. (See the Entering Data with an External Editing Program section below.) The list displays as a table with one row for each cell in the list. The first two lines of the table contain labels for the columns. These two lines of the table should not be modified when editing the data. The options for defining list data are shown on the right side of figure 3.

\section{Entering Parameters}

In MODFLOW-2005, a parameter is a single value that can be used to determine data values for multiple cells. Parameters often can make data input more convenient because of the multi-cell capability. By changing a single parameter value, the input data will be changed for all the associated cells. Also, input data must be defined using parameters in order to make use of parameter estimation. MFI2005 allows parameters to be defined for all the types of data for which parameters can be used in MODFLOW-2005.
The possibility for using parameters to define list data is shown on the left side of figure 3. List parameters can be used simultaneously with nonparameter lists. A list parameter is first created using the "New" pushbutton. A new list parameter is an empty list that can be populated. Values can be added to an existing list using the "Modify" pushbutton. When modifying a list parameter, the Package and Separate options can be used to specify the list. A list parameter can be active or inactive in any stress period and is controlled by the "Deactivate this stress period" and "Activate this stress period" pushbuttons. A common error is to create a parameter and not activate it in appropriate stress periods. List parameters can be deleted by using the "Delete" pushbutton.

A screen for defining arrays for the LPF Package is shown in figure 4 . The right side shows that the arrays can be defined without using parameters. The "Select Array" list shows the arrays that can be defined for the layer selected in the "Select layer" list. In this example, five array types can be defined for layer 1 . These arrays can also be defined using parameters, which are controlled by the left side of the screen. For any array type, all layers must be defined entirely with or without parameters. That is, parameters and nonparameters cannot be used to define the same array type. Once a parameter is defined that corresponds to an array type, MFI2005 removes that array type from the "Select Array" list of nonparameter arrays. Then, it is the user's responsibility to ensure that the parameters define data values for all cells for all layers for which that data type are required.

To define an array parameter, select the parameter type from the "Currently Allowed Parameter Types" list. The "New" pushbutton creates a new parameter as shown on the Array Parameter Definition screen (fig. 5). Each line in the table defines an array cluster, which defines values for all or part of the cells in one layer. The use of array clusters is

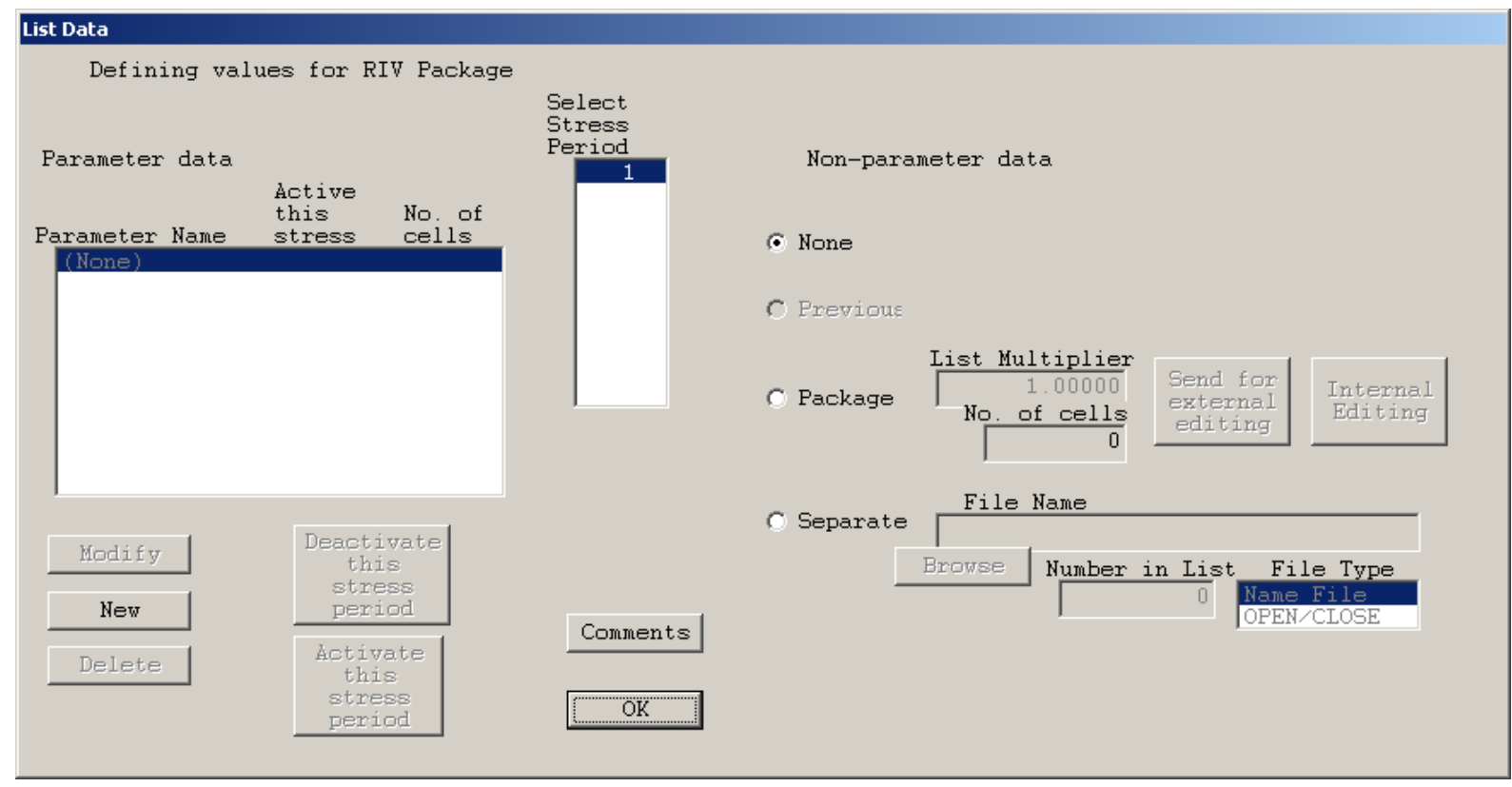

Figure 3. Example screen showing options for defining list data in MFI2005. 


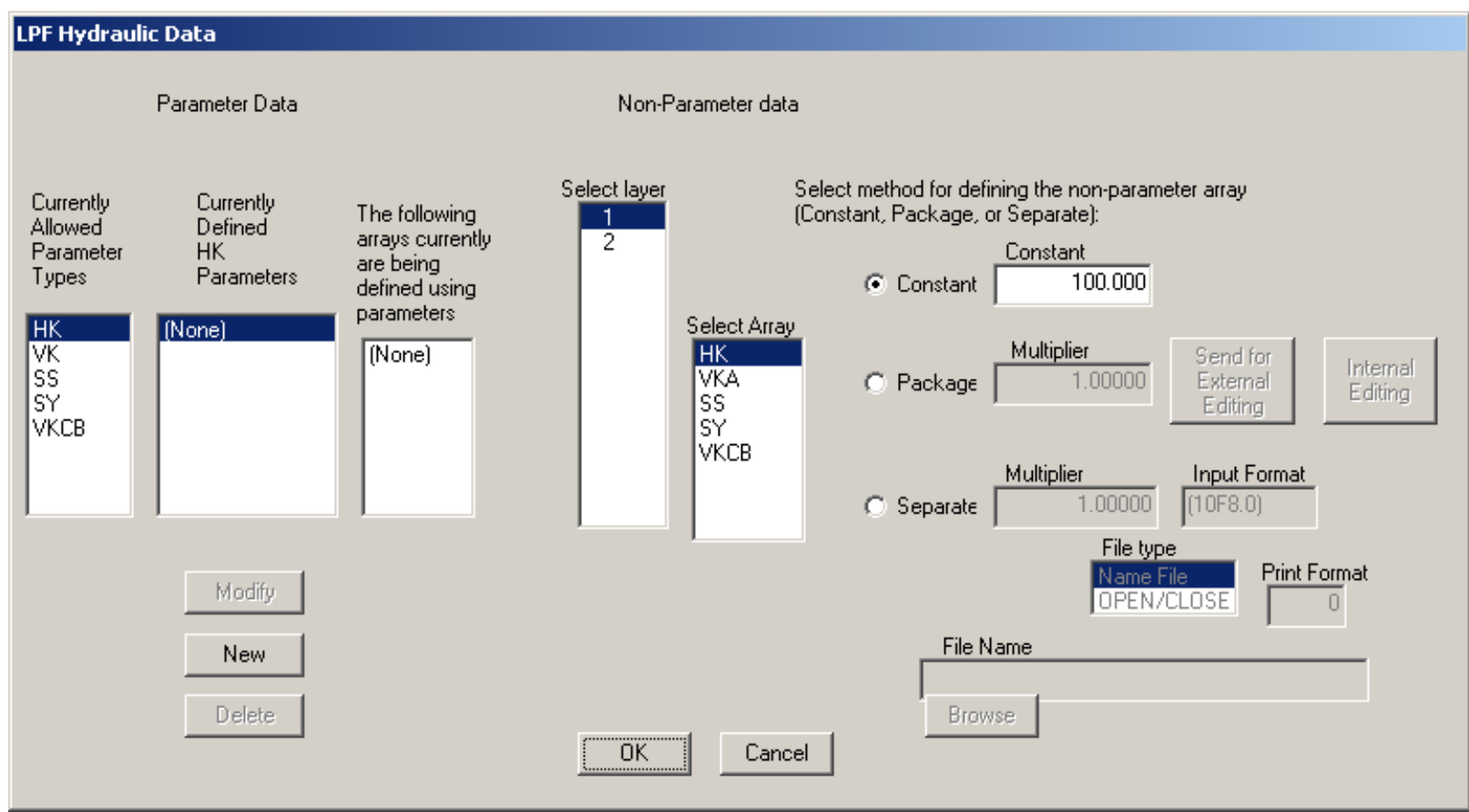

Figure 4. Example screen showing array definition in MFI2005.

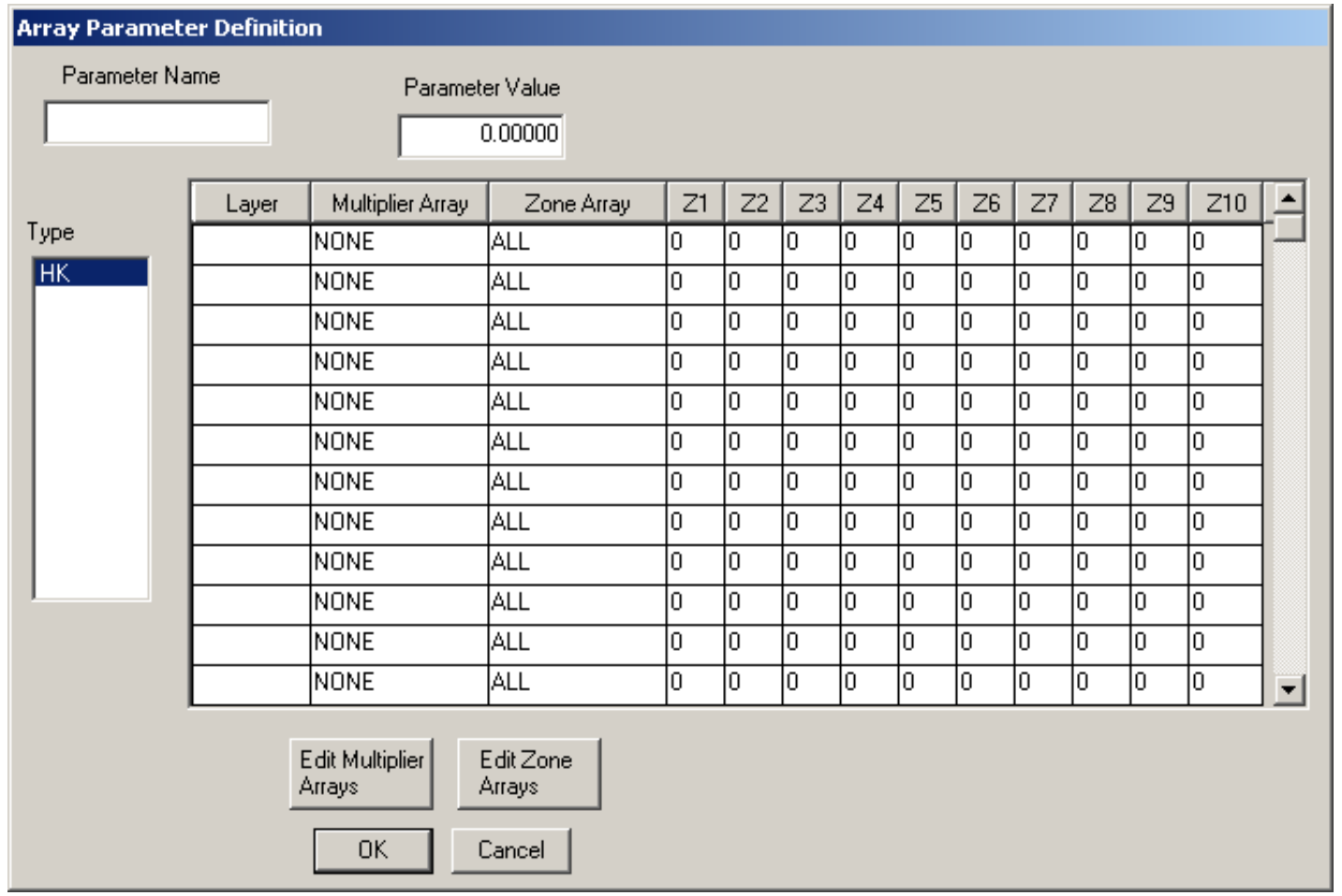

Figure 5. Example screen showing definition of array parameters in MFI2005. 
defined in Harbaugh (2005, p. 8-4 and 8-28). A zone array is used to specify which cells are associated with a parameter, and a multiplier array is used to scale the values for each cell. The value of "NONE" for the multiplier array indicates no scaling, and the value of "ALL" for a zone array indicates all cells in the layer. In order to use a multiplier array other than "NONE" or a zone array other than "ALL," the arrays must be defined. These arrays can be defined by selecting the ZoneMult menu item from the Main Menu or the "Edit Multiplier Arrays" and "Edit Zone Arrays" pushbuttons (fig. 5). When a zone array is used, 1 to 10 zone values must be specified in fields $Z 1$ through $Z 10$. If less than 10 zone values are used, the fields must be filled starting with Z1, and there must not be any blank fields between nonblank fields.

The "Modify" pushbutton on the array definition screen (fig. 4) allows an existing parameter to be modified using the array parameter screen (fig. 5). "The following arrays currently are being defined using parameters" list shows all array types that are being defined using parameters. To revert to defining an array type without using parameters, use the "Delete" pushbutton to delete all parameters of that type. The array type then will appear in the nonparameter data section of the screen in the "Select Array" list.

For LPF, the parameter types are the same as the array types, except for the VKA array. Parameters that define the VKA array are designated as VK or VANI depending on the value of the LAYVKA flags for each layer. The user must define appropriate parameter types to define VKA as specified by the LAYVKA flags (see Harbaugh, 2005, p. 5-20).

\section{OK and Cancel Pushbuttons}

As is typical in interactive programs, "OK" and "Cancel" pushbuttons are included on many of the MFI2005 screens. Both buttons cause the screen to be terminated, but the "Cancel" button causes data changes to be ignored, whereas "OK" causes changes to be accepted. However, the function of the "Cancel" button in MFI2005 is limited on many of the screens because some of the screen selections other than the "OK" pushbutton cause the current data to be accepted. Once acceptance occurs, "Cancel" can no longer prevent these data from being accepted. This action is easiest to explain by example. The screen that allows initial head to be specified (for the BAS Package) is shown in figure 2. In this example, the model consists of two layers. The screen contains a layer field, and the layer that currently is being defined is highlighted (layer 1 in fig. 2). In order to define data for a different layer, the desired layer can be selected. Changing the layer does not cause the screen to terminate but causes the data for the previous layer to be accepted. Thus, if the user changes from layer 1 to layer 2, the layer 1 data will be accepted. Thus, entering "Cancel" after changing to layer 2 will not prevent the changes that have been made to layer 1 from being accepted. Other screens allow more than one array to be defined as well as more than one layer. In these screens, changing the array also causes the current array to be accepted for the current layer.

\section{Obtaining Help When Using MFI2005}

For ease in identifying the specific data variables being entered, the MFI2005 data screens contain brief definitions of the variables being defined or specify the variable names (or both). For a more complete description of the data variables, the MODFLOW-2005 (Harbaugh, 2005), UCODE_2005 (Poeter and others, 2005), or MODPATH (Pollock, 1994, appendix A) documentation should be consulted. Further, the appendix contains help information for each screen. The organization of the information is similar to the MFI2005 Main Menu.

\section{Data Recovery if MFI2005 Aborts}

If something goes wrong and MFI2005 aborts, some data may be recoverable. If the error is detected by MFI2005, MFI2005 will attempt to save all current data in files named "MFI" followed by a period and a two- or three-character option designation; for example, "MFI.RCH" for the recharge option. Even if MFI2005 aborts without detecting the causefor example, the power to the computer is shut off-some data may be recoverable. Each time the menu for editing data for a primary option is completed, a backup file of the data for that option is written to an "MFI..." file. However, the user would have to use a text-editing program and have knowledge of the structure of the files (from MODFLOW-2005 or MODPATH documentation) to be able to extract data from the backup files.

\section{Entering Data with an External Editing Program}

MFI2005 allows the option of using an external editing program, such as a spreadsheet program, for entering array and list data. In a spreadsheet program, array values are displayed and edited as a table; the cell locations are designated implicitly by the row and column locations in the table. If the array must be entered for multiple layers, it is treated as multiple single-layer arrays. One-dimensional arrays consisting of one row of data or one column of data are displayed and edited in a spreadsheet program as a one-row or one-column table. For list data, the spreadsheet program will display a table with one line for location. Each line will include a layer, row, and column grid location plus one or more data values that apply at that location. If MODPATH is being used, each entry will also include a code to indicate the cell face through which the stress is applied.

The external editing program is run concurrently with MFI2005. When the external program is needed, MFI2005 writes the data to be edited into a file named_mfiss.csv and displays a screen instructing the user to activate the external program. The user must use the external program to read _mfiss.csv, edit the data, and save the data back into the same file. After the file has been closed within the external program, the user should select the OK pushbutton on the MFI2005 
screen, which will cause MFI2005 to read the file to obtain the edited data.

Users commonly encounter two problems with this approach. The first problem is that a user immediately selects $\mathrm{OK}$ on the screen that instructs the user to use the external program rather than waiting until the data have been edited. The second common problem is that a user fails to close mfiss.csv in the external program after saving the data. MFI2005 will be unable to read the edited values from the file because the file is still open in the external program. In this situation, MFI2005 displays a screen instructing the user to close the file in the external program.

MFI2005 writes the file for external editing ( csv) as a comma-delimited text file, and the external program must write the data back into the file using a comma-delimited structure. Excel and Lotus spreadsheet programs have been successfully used as external editing programs.

\section{Running MODFLOW-2005, MODPATH, and UCODE_2005}

MFI2005 creates a complete dataset for running MODFLOW-2005 and MODPATH except for the data files for arrays and lists that are specified using the Separate Method. The MODFLOW-2005 name file is fname.nam, where fname is the dataset name. If MODPATH is being used, MFI2005 also creates the MODPATH name file, and its name is fname.mpn.

MFI2005 also creates files needed to run UCODE_2005 if a MODFLOW-2005 Parameter Value File and at least one observation file are created. The Main UCODE_2005 input file is named fname_ucode.in, and the expected UCODE_2005 file prefix for creating files is fname ucode.

As noted above, the "Run MF2005" and "Run UCODE" commands in the FILE menu can be used to execute MODFLOW-2005 and UCODE_2005, respectively. In addition, MFI2005 creates Microsoft Windows ${ }^{\circledR}$ batch files to facilitate running MODFLOW-2005 and UCODE-2005 outside of MFI2005. The file named fname.bat will run the MODFLOW-2005 simulation if it is double-clicked in Windows Explorer ${ }^{\circledR}$. Similarly, the file named fname_ucode.bat will run the UCODE_2005 simulation if it is double-clicked in Windows Explorer ${ }^{\mathrm{B}}$. These ".bat" files include the pathnames for MODFLOW-2005 and UCODE_2005 specified in the "Pathname" command in the FILE menu. The required filename prefix for UCODE_2005 (Poeter and others, 2005, p. 29) is specified as fname_ucode.

Although MFI2005 creates datasets of the proper form for MODFLOW-2005, MODPATH, and UCODE_2005, incorrect data could possibly be entered using MFI2005. MFI2005 does some data checking in order to reduce the chance for errors, but the checking is not exhaustive. Further, no amount of checking can detect data-entry errors in which a reasonable, but incorrect, value is entered mistakenly. Some errors will cause MODFLOW-2005, MODPATH, or UCODE_2005 to stop before completion, whereas others will result in what might first appear to be normal execution, but the results are incorrect. Thus, the output files from MODFLOW-2005, MODPATH, and UCODE_2005 always should be examined to make sure that data have been defined as intended.

\section{Miscellaneous Details About Using MFI2005}

\section{Input Data that MFI2005 Generates}

Users already familiar with MODFLOW-2005 and MODPATH or who carefully examine the MODFLOW-2005 and MODPATH input instructions will notice that the MFI2005 data screens do not directly include all data variables required for MODFLOW-2005 and MODPATH. This is because MFI2005 generates some variables either by assumption or from other requested information. For example, the number of river reaches is determined by the number of nonblank lines in the list of river reaches specified in the file that is saved by the external editing program. File units are generated automatically.

\section{Input Data Shared Between MODFLOW and MODPATH}

Some variables are shared between MODFLOW and MODPATH. In MFI2005, these shared variables are entered only within MODFLOW data-editing screens. That is, the shared variables are not included as part of the screens used for MODPATH data. These shared variables are:

- HNOFLO

- HDRY

- LAYCON (BCF Package) or LAYTYP (LPF Package)

Note that IBOUND is not shared by MODPATH and MODFLOW, although both MODFLOW and MODPATH have an input variable named IBOUND. The reason is that MODPATH makes expanded use of IBOUND. In addition to indicating boundary conditions as in MODFLOW, MODPATH uses IBOUND to indicate zones for the purpose of controlling which particles and pathlines are displayed and their colors. Situations may occur in which different IBOUND values may be desired for MODFLOW and MODPATH. Thus, these are viewed as different arrays, except that when MODPATH is first activated, any MODFLOW IBOUND arrays that are constant or package arrays are copied to the IBOUND for MODPATH. Further, when IBOUND is modified in MODFLOW or MODPATH, the values are checked for consistency (no-flow, constant-head, and variable-head cells at the same locations). If there are inconsistencies, the user is notified, but nothing is done to attempt to remedy the inconsistencies. No consistency checking is done for any layers for which IBOUND is defined using the Separate Method in either MODFLOW or MODPATH. 
MODPATH includes the capability to specify which cell face receives a particular stress. MFI2005 supports this capability for the River, Well, Drain, and General-Head Boundary stresses through the screens in which stress data are entered. When MODPATH is active, these stress screens include an IFACE value for each location and indicate the cell face to which the stress is applied. Thus, activating MODPATH prior to entering stress data is more efficient than waiting until after stress data are already defined. This specification will allow the stress faces to be entered when the other stress data are entered. If stress data are entered prior to activating MODPATH, the stress screens will need to be reentered after MODPATH is activated if there is a need to specify which faces are to receive stresses. For Recharge and Evapotranspiration stresses, the face is specified as a single value for all cells for the entire simulation. This value is specified in the Misc. option of the MODPATH menu.

Note that for the IFACE data to be conveyed from MODFLOW-2005 to MODPATH, the COMPACT BUDGET option must be specified on the "Output Files and Formats" screen that is accessed through the "Output" Main Menu item.

\section{Constraints in MFI2005 That Are Not in MODFLOW-2005 and MODPATH}

Ideally, MFI2005 should impose no constraints on data that MODFLOW-2005 and MODPATH do not impose; however, some compromises were made to minimize development effort. Additional constraints imposed by MFI2005 are listed below. Some configurable limits were set in the program, which relate to the amount of memory used by MFI2005 and are not included in this list of constraints.

1. The precision of data values in files created by MFI2005 is controlled by MFI2005. In most cases, values will be written with at least four significant digits. If the precision used is inadequate, MFI2005 cannot be used or must be modified to be more precise.

2. If output does not occur at regular intervals (every time step, end of every stress period, or end of simulation), then a maximum of 47 times can be entered at which output will occur.

3. If specific layers for printing or saving head or drawdown are listed rather than using the default of having this information output for all layers, a maximum of 15 layers can be specified.

4. A maximum of 92 file units can be used for MODFLOW files - units 1-4 and 7-94. A maximum of 77 file units can be used for MODPATH files - units 1-4 and 7-79.

\section{Using MFI2005 When MODFLOW-2005 Includes Capabilities That MFI2005 Does Not Include}

MODFLOW-2005 is designed to be enhanced, and accordingly, MFI2005 generally will not be up-todate with MODFLOW-2005. If a user is using parts of MODFLOW-2005 that are not supported by MFI2005, the program still can be used for those parts that MFI2005 does support. Files for packages not supported by MFI2005 must be prepared by other means, and the names of these files must be added manually to the name file. If MFI2005 does not support a file type that is included in the name file, MFI2005 will leave the line containing the unknown file type in the MODFLOW-2005 Name File.

\section{UCODE_2005 Files}

MFI2005 creates files needed to run UCODE 2005 if a MODFLOW_2005 Parameter Value File and at least one observation file are created. First, a main UCODE_2005 input file is created. The main UCODE_2005 input file is named fname_ucode.in. The UCODE_2005 dialog is entered by selecting the UCODE Main Menu item.

A UCODE_2005 parameter file, named fname_ucode. par, is created, and the data are entered in the Parameter Value dialog. The StartValue, LowerValue, UpperValue, ScalePval, Transform, Adjustable, Constrain, LowerConstraint, and UpperConstraint fields are not used for MODFLOW_2005 files. All of these values are documented in the UCODE_2005 documentation.

A UCODE_2005 observation file, named fname_ucode. obs, is created, and the data are entered in the observation dialogs. The Statistic, StatFlag, and PlotSymbol fields are not used for MODFLOW-2005 files. The PlotSymbol field is made to be the UCODE_2005 PlotSymbol using the UCODE_2005 group capability. A UCODE_2005 Observation_Groups Input Block is created in the main UCODE input file, and this block assigns the PlotSymbol to be the same as the GroupName.

A UCODE_2005 template file, named fname_ucode. tpl, is created. This template file describes how to generate a MODFLOW-2005 Parameter Value file so that UCODE_2005 can run MODFLOW-2005 with different parameter values to compute parameter sensitivities.

A UCODE_2005 instruction file, named fname_ucode. inst, is further created. This file describes how to read the simulated equivalents for observations produced by MODFLOW-2005. The values produced by MODFLOW-2005 must all be written into the same file, which is specified in the observation dialogs.

MFI2005 makes an additional MODFLOW-2005 Name File, which UCODE_2005 uses to run MODFLOW-2005. 
This file is named fname.nam.ucode. This additional Name File is identical to fname.nam except that the Parameter Value file is named ucode.pvl rather than the user-defined name. The reason for the change is to avoid having UCODE_2005 overwrite the Parameter Value file when UCODE_2005 runs. UCODE_2005 recreates the ucode.pvl Parameter Value file many times as it computes sensitivities of parameters.

MFI2005 further creates a special file named fname.mfi that MFI2005 uses to save data needed to create UCODE files. Although the data in this file can be found in various places in the UCODE files, MFI2005 has not been programmed to read UCODE files.

\section{Using MFI2005 With a Dataset Not Originally Created by MFI2005}

MFI2005 can work with MODFLOW-2005 and MODPATH files that were not originally created using MFI2005. The main constraint is that the name files must end in .nam and .mpn, respectively. Also, it should be noted that MFI2005 will not rewrite the data in the same format as previously used. Thus, some precision of values may be lost. As described above, MFI2005 cannot read UCODE files, and accordingly, MFI2005 is not able to use UCODE files that were created outside of MFI2005.

\section{Program Documentation}

The source code for MFI2005 is not included in this report; the code can be obtained from the Internet as described in the report Preface. The information in the following section is provided to help the user modify the source code if needed.

\section{Memory Usage}

MFI2005 stores in memory the data that are read. MFI2005 uses a single array, X, to store most of its data. MFI2005 maintains a list of variable names and their locations within X. The list is searched when the location of a variable is needed.

Lists of stress data for a stress period require special consideration because MFI2005 does not require the maximum size of a list to be specified. MFI2005 allows the user to enter list data of varying length, and the length can change at any time. In order to store lists of varying size, MFI2005 allocates memory for lists in blocks. Each block has a fixed size, but a list can consist of up to 10 blocks.

The following variables control how much memory MFI2005 can use. These variables are defined in the file "MFI2005.inc," which is included in many of the subroutines of MFI2005. In addition to the size of the X array, these variables specify the size of a number of other arrays used by MFI2005 to keep track of data.

- LENX - Number of elements in the X array

- MXVAR-Maximum number of variables

- MXARR-Maximum number of variables that are arrays

- MXFNAM-Maximum number of files

- MXLIST-The number of entries in a list block; a list can consist of up to 10 blocks

\section{Portability}

Although MFI2005 is written in Fortran, which has been widely implemented on many kinds of computers, MFI2005 is strongly dependent on the Winteracter Subroutine Library (Interactive Software Services, 2003). Winteracter is a commercial product that is not as widely available as Fortran. Calls to the Winteracter Subroutine Library are dispersed throughout the parts of the program that interact with users.

\section{Summary}

Computer models of groundwater flow require input data to define the simulated flow system. The MFI2005 datainput program was developed by the U.S. Geological Survey (USGS) for use with the modular three-dimensional finitedifference groundwater model, MODFLOW-2005. MFI2005 is designed to be easy to use and to run on personal computers. Data are entered interactively through a series of display screens. MFI2005 also supports the UCODE_2005 parameterestimation program and the MODPATH particle-tracking program. Other data-entry programs can be used in conjunction with MFI2005. MFI2005 can be downloaded from the Internet (see report Preface), and user instructions are provided. 


\section{References Cited}

Banta, E.R., 2006, Modifications to MODFLOW boundary conditions and an adaptive-damping scheme for Picard iterations for a highly nonlinear regional model, in Poeter, Eileen, Hill, Mary, and Zheng, Chunmiao, Proceedings, MODFLOW and More 2006-Managing Ground Water Systems: International Ground Water Modeling Center and Colorado School of Mines, Golden, Colorado, 2006, p. 596-600.

Goode, D.J., and Appel, C.A., 1992, Finite-difference interblock transmissivity for unconfined aquifers and for aquifers having smoothly varying transmissivity: U.S. Geological Survey Water-Resources Investigations Report 92-4124, 79 p.

Harbaugh, A.W., 1995, Direct solution package based on alternating diagonal ordering for the U.S. Geological Survey modular finite-difference ground-water flow model: U.S. Geological Survey Open-File Report 95-288, 46 p.

Harbaugh, A.W., 2002, A data input program (MFI2K) for the U.S. Geological Survey modular ground-water model (MODFLOW-2000): U.S. Geological Survey Open-File Report 02-41, $55 \mathrm{p}$.

Harbaugh, A.W., 2005, MODFLOW-2005, The U.S. Geological Survey modular ground-water model-The groundwater flow process: U.S. Geological Survey Techniques and Methods 6-A16, variously paged.

Harbaugh, A.W., Banta, E.R., Hill, M.C., and McDonald, M.G., 2000, MODFLOW-2000, the U.S. Geological Survey modular ground-water model-User guide to modularization concepts and the ground-water flow process: U.S. Geological Survey Open-File Report 00-92, 121 p.
Hill, M.C., 1990, Preconditioned conjugate-gradient 2 (PCG2), a computer program for solving ground-water flow equations: U.S. Geological Survey Water-Resources Investigations Report 90-4048, 43 p.

Hill, M.C., 1998, Methods and guidelines for effective model calibration: U.S. Geological Survey Water-Resources Investigations Report 98-4005, 90 p.

Interactive Software Services, 2003, Winteracter 5.0 Subroutine Reference: Interactive Software Services Ltd.

Mehl, S.W., and Hill, M.C., 2001, MODFLOW-2000, the U.S. Geological Survey modular ground-water modelUser guide to the Link-AMG (LMG) Package for solving matrix equations using an algebraic multigrid solver: U.S. Geological Survey Open-File Report 01-177, 33 p.

Poeter, E.P., Hill, M.C., Banta, E.R., Mehl, Steffen, and Christensen, Steen, 2005, UCODE_2005 and six other computer codes for universal sensitivity analysis, calibration, and uncertainty evaluation: U.S. Geological Survey Techniques and Methods 6-A11, $283 \mathrm{p}$.

Pollock, D.W., 1994, User's guide for MODPATH/ MODPATH-PLOT, Version 3-A particle tracking postprocessing package for MODFLOW, the U.S. Geological Survey finite-difference ground-water flow model: U.S. Geological Survey Open-File Report 94-464, variously paged.

Wilson, J.D., and Naff, R.L., 2004, MODFLOW-2000, the U.S. Geological Survey modular ground-water modelGMG Linear Equation Solver Package documentation: U.S. Geological Survey Open-File Report 2004-1261, 47 p. 


\section{Appendix-MFI2005 Help Information}

This appendix contains help information for each screen. The information is organized like the MFI2005 Main Menu. References cited in this appendix are listed in the References Cited section of the report. Although this help information is more complete than the help provided on the MFI2005 screens, additional information still may be required. The user should consult the appropriate MODFLOW-2005 or MODPATH documentation for additional information.

\section{File}

\section{Open}

A new dataset is opened. The user is prompted to choose whether or not to save the currently open dataset.

\section{Save As}

The dataset is saved using a new name, and MFI2005 continues editing the new dataset. The old dataset is left as is.

\section{Save}

The dataset is saved using the current name. The old dataset is overwritten.

\section{Pathnames}

Pathnames for the MODFLOW-2005 and UCODE_2005 executable files can be specified. The names can be simple file names if the files are located within a directory in the Windows ${ }^{\circledR}$ search path. For example, the default pathname for MODFLOW-2005 is MF2005. This default is sufficient if the MODFLOW-2005 executable is named MF2005.EXE and is located in any of the directories that are specified in the search path. The pathnames are saved in the ".mfi" file for each dataset; therefore, the pathnames must be individually specified for each dataset.

\section{Run MF2005}

The current dataset is saved, and MODFLOW-2005

is executed.

\section{Run UCODE}

The current dataset is saved, and UCODE_2005 is executed.

\section{Abort}

MFI2005 is exited without saving the data. Any changes made to the data since a "Save As," "Save," "Run MF2005," or "Run UCODE" menu item was selected will be lost. The user will be prompted to determine if this is the intent.

\section{Exit (and Save)}

The dataset is saved and MFI2005 is exited. If some data are incomplete, the user is prompted and allowed to go back to the main menu. If the user chooses to exit with incomplete data, MFI2005 will allow the user to complete data editing the next time the dataset is edited.

\section{Options}

\section{Check Complete}

All of the active MODFLOW simulation options are displayed, and a status flag is shown for each option that indicates whether or not data entry is complete. For any option that is incomplete, the specific missing data can be displayed by using the Check pushbutton on the data-editing screen where the data for that MODFLOW option is entered.

\section{Deactivate}

This option deactivates (turns off) MODFLOW options. Using a data-editing screen automatically turns on the corresponding option. For example, selecting RCH in the STRESS menu turns on RCH (recharge). The Deactivate option allows recharge to be turned off.

\section{Show Files}

The names of all files that are being used for the MODFLOW simulation are displayed.

\section{LIST File}

This option allows the name of the Listing File to be specified.

\section{Zone-Mult}

\section{Zone Arrays}

The zone file defines two-dimensional arrays that are used to define array parameters.

The "Select Zone Array" list allows a zone array to be selected for editing. The following options allow the values of the array to be defined:

- Constant - all elements of the array have the same value.

- Package - data are saved in the zone file, and the internal or external editing mechanism is used to modify the data.

- Separate - data are stored in a separate file that MFI2005 does not read.

The "ADD" pushbutton allows a new zone array to be created.

The "Delete" pushbutton causes the currently selected zone array to be deleted.

The "Comments" pushbutton allows comments to be defined. These comments are placed at the beginning of the zone file. 


\section{Multiplier Arrays}

The multiplier file defines two-dimensional arrays that are used to define array parameters. MFI2005 does not allow multiplier-array functions to be edited; however, if an existing dataset includes functions, these are maintained when MFI2005 saves the dataset.

The "Select Multiplier Array" list allows a multiplier array to be selected for editing. The following editing options allow the values of each array element to be defined:

- Constant - all elements of the array have the same value.

- Package - data are saved in the multiplier file, and the internal or external editing mechanism is used to modify the data.

- Separate - data are stored in a separate file that MFI2005 does not read.

The "ADD" pushbutton allows a new multiplier array to be created.

The "Delete" pushbutton causes the currently selected multiplier array to be deleted.

The "Comments" pushbutton allows comments to be defined. These comments are placed at the beginning of the multiplier file.

\section{DIS}

Discretization File. For the input variables that are arrays, the following editing options allow the values of each array element to be defined:

- Constant - all elements of the array have the same value.

- Package - data are saved in the DIS file, and the internal or external editing mechanism is used to modify the data.

- Separate — data are stored in a separate file that MFI2005 does not read.

\section{Check}

This option checks the DIS data to determine whether data are complete. If not complete, the incomplete data are listed.

\section{Time and Length Units}

The time unit of model data must be consistent for all data values that involve time. For example, if years is the chosen time unit, stress-period length, time-step length, transmissivity, and so on, all must be expressed using years for their time units. Note that the program still will run even if "undefined" time units are specified because the fundamental equations used in
MODFLOW do not require that the time unit be identified. Consistent units should be used for all input data even when ITMUNI indicates an undefined time unit. When the time unit is defined, MODFLOW uses the time unit to print a table of elapsed simulation time. The acceptable values are:

$\begin{array}{ll}\text { Undefined } & \text { Hours } \\ \text { Seconds } & \text { Days } \\ \text { Minutes } & \text { Years }\end{array}$

The length unit of model data must be consistent for all data values that involve length. For example, if feet is the chosen length unit, grid spacing, head, hydraulic conductivity, water volumes, and so forth, all must be expressed using feet for their length units. Note that the program still will run even if "undefined" length units are specified because the fundamental equations used in MODFLOW do not require that the length unit be identified. Consistent units should be used for all input data even when LENUNI indicates an undefined length unit. The acceptable values are:

$$
\begin{aligned}
& \text { Undefined } \\
& \text { Feet } \\
& \text { Meters } \\
& \text { Centimeters }
\end{aligned}
$$

\section{DELR}

DELR is the cell width along rows. Read one value for each of the NCOL columns. DELR is a one-dimensional variable with one value for each column.

\section{DELC}

DELC is the cell width along columns. Read one value for each of the NROW rows. DELC is a one-dimensional variable with one value for each row.

\section{LAYCBD}

LAYCBD indicates whether or not a layer has a Quasi-3D confining bed below it. "No" indicates no confining bed, and "Yes" indicates a confining bed. LAYCBD for the bottom layer must be "No."

\section{TOP}

Top is the top elevation of layer 1 and is a two-dimensional array. For the common situation in which the top layer represents a water-table aquifer, setting the value for Top equal to land-surface elevation may be reasonable.

\section{BOTM}

BOTM is the bottom elevation of a model layer or a Quasi-3D confining bed. BOTM is a set of two-dimensional arrays. 


\section{Stress Periods}

"Number of Stress Periods" is the number of stress periods in the simulation (variable NPER).

"Length" is the length of a stress period (variable PERLEN).

"Steps" is the number of time steps in a stress period (variable NSTP).

"Multiplier" is the multiplier for the length of successive time steps (variable TSMULT). The length of a time step is calculated by multiplying the length of the previous time step by TSMULT. The length of the first time step, $\Delta \mathrm{t}_{1}$, is related to PERLEN, NSTP, and TSMULT by the relation:

$\Delta \mathrm{t}_{1}=$ PERLEN $\left(\mathrm{TSMULT}^{-1}\right) /\left(\mathrm{TSMULT}^{\mathrm{NSTP}}-1\right)$

$\mathrm{Ss} / \mathrm{tr}$ is a character variable that indicates whether the stress period is steady state or transient. The only allowed options are "SS" or "TR," and the characters are case insensitive.

\section{Comments}

Comments are placed at the beginning of the DIS file.

\section{BAS}

\section{Basic Package.}

\section{Check Data}

This option checks the BAS Package data to determine whether data are complete. If not complete, the incomplete data are listed.

\section{Miscellaneous}

The "Comments" field allows comments, which are placed at the beginning of the BAS Package file to be defined. The first two comment lines will become variable HEADNG, which is used as a printout title throughout the program. (If there are no comment lines, then HEADNG will be blank.) HEADNG is limited to 80 columns, but subsequent Text lines can be up to 199 columns. Any character can be included in Text. The "\#” character must be in column 1 .

HNOFLO is the value of head to be assigned to all inactive (no flow) cells $($ IBOUND $=0$ ) throughout the simulation. Because head at inactive cells is unused in model calculations, this does not affect model results but serves to identify inactive cells when head is printed. This value also is used as drawdown at inactive cells if the drawdown option is used. Even if the user does not anticipate having inactive cells, a value for HNOFLO must be entered.

Format flag indicates whether fixed or free format is used for input variables throughout the Basic Package and other packages as indicated in their input instructions.
IBOUND

IBOUND is the boundary variable. One value is read for every model cell. Usually, these values are read a layer at a time; however, when the XSECTION option is specified, a single array for the cross section is read. Note that although IBOUND is read as one or more two-dimensional variables, it is stored internally as a three-dimensional variable.

If IBOUND $(\mathrm{J}, \mathrm{I}, \mathrm{K})<0$, cell $\mathrm{J}, \mathrm{I}, \mathrm{K}$ has a constant head.

If $\operatorname{IBOUND}(\mathrm{J}, \mathrm{I}, \mathrm{K})=0$, cell $\mathrm{J}, \mathrm{I}, \mathrm{K}$ is inactive (no flow).

If IBOUND $(J, I, K)>0$, cell J,I,K is active (variable head).

The following editing options allow the IBOUND values to be defined for each layer:

- Constant-all elements of the array have the same value.

- Package - data are saved in the BAS file, and the internal or external editing mechanism is used to modify the data.

- Separate - data are stored in a separate file that MFI2005 does not read.

\section{Initial Head}

Initial head is the head at the beginning of the simulation, which is variable STRT. STRT must be specified for all simulations, including steady-state simulations. One value is read for every model cell. Usually, these values are read one layer at a time. When the XSECTION option is specified, however, a single array for the cross section is read. For simulations in which the first stress period is steady state, the values used for STRT generally do not affect the simulation [exceptions may occur if cells go dry and (or) rewet]. The execution time, however, will be less if STRT includes hydraulic heads that are close to the steady-state solution.

The following editing options allow the STRT values to be defined for each layer:

- Constant-all elements of the array have the same value.

- Package - data are saved in the BAS file, and the internal or external editing mechanism is used to modify the data.

- Separate - data are stored in a separate file that MFI2005 does not read. 


\section{Internal Flow-BCF and LPF}

The Block-Centered Flow (BCF) Package and Layer-Property Flow (LPF) Package are alternative internal flow packages. Both packages cannot be used simultaneously. If one package is active and the other package is selected, the user is prompted to see if it is OK to deactivate the package that is already active.

\section{BCF-Block-Centered Flow Package}

\section{Check Data}

This option causes MFI2005 to check the BCF Package data to determine if data are complete. If not, the incomplete data are listed.

\section{Miscellaneous}

Head at cells that go dry (variable HDRY) is the head that is assigned to cells that are converted to dry during a simulation. Although this value is not used in the model calculations, HDRY is useful as an indicator when looking at the resulting heads that are output from the model. HDRY is, thus, similar to HNOFLO in the Basic Package, which is the value assigned to cells that are no-flow cells at the start of a model simulation.

OFF and ON Wetting Capability options-OFF indicates that wetting is turned off, and $\mathrm{ON}$ indicates that wetting is turned on.

WETFCT is a factor that is included in the calculation of the head that initially is established at a cell when the cell is converted from dry to wet. (See IHDWET.)

IWETIT is the iteration interval for attempting to wet cells. Wetting is attempted for every IWETIT iteration. If using the PCG solver (Hill, 1990), this value applies to outer iterations, not inner iterations. If IWETIT is 0 , the value is changed to 1 .

IHDWET is an option that determines which equation is used to define the initial head at cells that become wet:

$$
\text { BOT }+ \text { WETFCT }\left(h_{n}-\text { BOT }\right)
$$$$
\text { BOT + WETFCT (THRESH) }
$$

\section{LAYCON}

The LAYCON option activates a screen that is used to specify three values for each layer of the model grid:

LAYCON is the layer type:

- Confined-Transmissivity and storage coefficient of the layer are constant for the entire simulation $(\mathrm{LAYCON}=0)$.

- Unconfined-Transmissivity of the layer varies $(\mathrm{LAYCON}=1)$ and is calculated from the saturated thickness and hydraulic conductivity. The storage coefficient is constant. This type code is valid only for layer 1.
- Partially Convertible-Transmissivity of the layer is constant, but the storage coefficient may alternate between confined and unconfined values $(\mathrm{LAYCON}=2)$. Vertical flow from above is limited if the layer desaturates.

- Fully Convertible-Transmissivity of the layer varies, and the storage coefficient may alternate between confined and unconfined values ( $\mathrm{LAYCON}=3$ ). Transmissivity is calculated from the saturated thickness and hydraulic conductivity. Vertical flow from above is limited if the layer desaturates.

Horizontal Anisotropy (Variable TRPY) is the ratio of transmissivity or hydraulic conductivity (whichever is being used for input) in the $\mathrm{Y}$ direction (along a column) to transmissivity or hydraulic conductivity in the $\mathrm{X}$ direction (along a row). Specify 1.0 for isotropic layers.

LAYAVG defines the method of calculating interblock transmissivity. The methods are described by Goode and Appel (1992).

- Harmonic $(\mathrm{LAYAVG}=0)$-Harmonic mean

- Arithmetic $(\mathrm{LAYAVG}=1)$-Arithmetic mean

- Logarithmic (LAYAVG = 2)-Logarithmic mean

- Arith-log (LAYAVG = 3)-Arithmetic mean of saturated thickness and logarithmic-mean hydraulic conductivity.

\section{Hydraulic Data}

This screen allows hydraulic arrays to be entered for each layer as required according to the options in effect such as steady state versus transient, wetting on or off, and layer type. The following editing options allow the array values to be defined for each layer:

- Constant-all elements of the array have the same value.

- Package - data are saved in the BCF file, and the internal or external editing mechanism is used to modify the data.

- Separate - data are stored in a separate file that MFI2005 does not read.

Sfl is the primary storage coefficient. Read only if there are one or more transient stress periods specified in the Discretization File. For LAYCON equal to 1, Sfl always will be specific yield, whereas for LAYCON equal to 2 or $3, \mathrm{Sf} 1$ always will be confined storage coefficient. For LAYCON equal to $0, \mathrm{Sf} 1$ would normally be confined storage coefficient; however, a LAYCON value of 0 also can be used to simulate unconfined conditions where drawdowns are expected to remain everywhere a small fraction of the saturated thickness, and where there is no layer above, or flow from above is negligible. In this case, specific yield values would be entered for Sf1. 
Tran is the transmissivity along rows. Tran is multiplied by TRPY to obtain transmissivity along columns. Read only for layers where LAYCON is 0 or 2.

HY is the hydraulic conductivity along rows. HY is multiplied by TRPY to obtain hydraulic conductivity along columns. Read only for layers where LAYCON is 1 or 3.

Vcont is the vertical hydraulic conductivity divided by the thickness of a layer to the layer below. The value for a cell is the hydraulic conductivity divided by thickness for the material between the node in that cell and the node in the cell below. Because there is no layer beneath the bottom layer, Vcont cannot be specified for the bottom layer.

$\mathrm{Sf} 2$ is the secondary storage coefficient. Read only for layers where LAYCON is 2 or 3 and only if there are one or more transient stress periods specified in the Discretization File. The secondary storage coefficient always is specific yield.

WETDRY is a combination of the wetting threshold (THRESH) and a flag to indicate which neighboring cells can cause a cell to become wet. If WETDRY $<0$, only the cell below a dry cell can cause the cell to become wet. If WETDRY $>0$, the cell below a dry cell and the four horizontally adjacent cells can cause a cell to become wet. If WETDRY is 0 , the cell cannot be wetted. The absolute value of WETDRY is the wetting threshold. When the sum of BOT and the absolute value of WETDRY at a dry cell is equaled or exceeded by the head at an adjacent cell, the cell is wetted. Read only if LAYCON is 1 or 3 and IWDFLG is not 0 .

\section{LPF_Layer-Property Flow Package}

\section{Check}

This option causes MFI2005 to check the LPF Package data to determine if data are complete. If not complete, the incomplete data are listed.

\section{Miscellaneous}

Head assigned to cells that convert to dry (variable HDRY) is the head that is printed or saved at cells that have converted to dry during a simulation. Although this value is not used in the model calculations, it is useful as an indicator when looking at the resulting heads that are output from the model. HDRY is, thus, similar to HNOFLO in the Basic Package, which is the value assigned to cells that are no-flow cells at the start of a model simulation.

STORAGECOEFFICIENT option indicates that the Ss variable and SS parameters are read as storage coefficient rather than specific storage.

CONSTANTCV option indicates that vertical conductance for an unconfined cell is computed from cell thickness rather than saturated thickness.
THICKSTRT option indicates that layers where the LAYTYP flag is THISKSTRT are confined and have their cell thickness for conductance calculations computed from STRT-BOT rather than TOP-BOT.

NOCVCORRECTION option indicates that vertical conductance is not corrected when the vertical flow correction is applied for a perched water table.

NOVFC option indicates that the vertical flow correction for a perched water table is totally turned off.

Factor that affects initial head when cells become wet (WETFCT) is a variable that is included in the equation for the head that initially is established at a cell when it is converted from dry to wet. (See IHDWET.)

Iteration interval for attempting to wet cells (IWETIT) determines the iterations at which wetting is attempted at wettable cells. Wetting is attempted for every IWETIT iteration. If using the PCG solver (Hill, 1990), this value applies to outer iterations, not inner iterations. If IWETIT is 0 , it is changed to 1 .

Equation to define initial head when cells become wet (IHDWET) is an option that determines which equation defines the initial head at cells that convert from dry to wet:

$$
\begin{aligned}
& \text { BOT + WETFCT }\left(h_{n}-\text { BOT }\right) \\
& \text { BOT + WETFCT }(\text { THRESH })
\end{aligned}
$$

\section{LAYTYP}

The LAYTYP option activates a screen that is used to specify five values for each layer of the model grid:

1. LAYTYP is the layer type:

- Confined-Transmissivity and storage coefficient of the layer are constant for the entire simulation $($ LAYTYP $=0)$.

- Convertible-Transmissivity of the layer varies, and the storage coefficient may alternate between confined and unconfined values (LAYTYP $\neq 0$ ). Transmissivity is calculated from the saturated thickness and hydraulic conductivity. Vertical flow from above is limited if the layer desaturates. For the water table, specify convertible and be sure that the top elevation is higher than the computed head.

- THICKSTRT-The layer is confined (transmissivity and storage coefficient are constant for the entire simulation), and the thickness of the layer comes from STRT minus Bottom. This option can be set only if the THICKSTRT option is specified in the Miscellaneous Menu. 
2. LAYAVG defines the method of calculating interblock transmissivity. The methods are described by Goode and Appel (1992).

- Harmonic (LAYAVG $=0$ ) - -Harmonic mean (the method used in MODFLOW-88).

- Logarithmic (LAYAVG = 1)—Logarithmic mean.

- Arith-log (LAYAVG = 2)-Arithmetic mean of saturated thickness and logarithmic-mean hydraulic conductivity.

3. CHANI (horizontal anisotropy) is the ratio of hydraulic conductivity in the $\mathrm{Y}$ direction (along a column) to hydraulic conductivity in the $\mathrm{X}$ direction (along a row). A nonzero value here applies to all cells in the layer. Specify 1.0 for isotropic layers. Specify 0.0 in order to use array HANI to specify individual values for every cell in the layer through the Hydraulic Data screen.

4. LAYVKA is a flag indicating whether array VKA is vertical hydraulic conductivity or the ratio of horizontal to vertical hydraulic conductivity.

5. LAYWET is the wetting flag. Wetting can be on or off for each layer. Wetting cannot be on for a confined layer.

\section{Hydraulic Data}

This screen allows hydraulic arrays to be entered for each layer as required according to the options in effect such as steady state versus transient, wetting on or off, and layer type. Some of these arrays can be defined using the following parameters:

- HK is the parameter type for defining array HK.

- VK is the parameter type for defining VKA when VKA is vertical hydraulic conductivity.

- VANI is the parameter type for defining VKA when VKA is the ratio of horizontal to vertical hydraulic conductivity.

- SS is the parameter type for defining array Ss.

- SY is the parameter type for defining array Sy.

- $\mathrm{VKCB}$ is the parameter type for defining array VKCB.

- HANI is the parameter type for defining array HANI.

If an array is defined using parameters, then all values of the array must be defined using parameters. The parameters are defined by specifying a parameter name, parameter type, parameter value, and one or more clusters that specify the layer, multiplier array, zone array, and zone codes that are associated with the parameter.
If parameters are not used to define an array, then the values are defined as two-dimensional arrays for each layer in which the array is required. The following editing options allow the array values to be defined for each layer:

- Constant - all elements of the array have the same value.

- Package - data are saved in the LPF file, and the internal or external editing mechanism is used to modify the data.

- Separate - data are stored in a separate file that MFI2005 does not read.

The arrays that are being defined as two-dimensional arrays are shown in the "Select Array" list. Depending on the options specified in the LAYTYP screen and the arrays being defined as parameters, the possible arrays in the "Select Array" list are:

$\mathrm{HK}$ is the hydraulic conductivity along rows. HK is multiplied by HANI or CHANI to obtain hydraulic conductivity along columns.

HANI is the ratio of hydraulic conductivity along columns (Y direction) to the hydraulic conductivity along rows (X direction). This array is only specified for layers in which CHANI is 0.0 .

VKCB is the vertical hydraulic conductivity of a Quasi 3 -D confining bed. This array is only defined for layers that are underlain by a Quasi-3D confining bed.

VKA is the vertical hydraulic conductivity or the ratio of horizontal hydraulic conductivity (HY) to vertical hydraulic conductivity. The type of data depends on the value of LAYVKA for each layer.

Ss is the specific storage. This array is only required when there is at least one transient stress period.

Sy is the specific yield. This array is only required when there is at least one transient stress period and for layers that are convertible.

WETDRY is a combination of the wetting threshold (THRESH) and a flag to indicate which neighboring cells can cause a cell to become wet. If WETDRY $<0$, only the cell below a dry cell can cause the cell to become wet. If WETDRY $>0$, the cell below a dry cell and the four horizontally adjacent cells can cause a cell to become wet. If WETDRY is 0 , the cell cannot be wetted. The absolute value of WETDRY is the wetting threshold. When the sum of BOT and the absolute value of WETDRY at a dry cell is equaled or exceeded by the head at an adjacent cell, the cell is wetted. This array is required only for layers that are wettable. 


\section{Print Codes}

This option allows print codes for each layer to be specified for use with parameters. The print codes specify the format for printing the resulting input values after the values are created from parameters. If any array is not determined from parameters, then these print codes have no effect on print format. The codes are:

$\begin{array}{ll}0-10 \mathrm{G} 11.4 & 11-20 \mathrm{~F} 5.4 \\ 1-11 \mathrm{G} 10.3 & 12-10 \mathrm{G} 11.4 \\ 2-9 \mathrm{G} 13.6 & 13-10 \mathrm{~F} 6.0 \\ 3-15 \mathrm{~F} 7.1 & 14-10 \mathrm{~F} 6.1 \\ 4-15 \mathrm{~F} 7.2 & 15-10 \mathrm{~F} 6.2 \\ 5-15 \mathrm{~F} 7.3 & 16-10 \mathrm{~F} 6.3 \\ 6-15 \mathrm{~F} 7.4 & 17-10 \mathrm{~F} 6.4 \\ 7-20 \mathrm{~F} 5.0 & 18-10 \mathrm{~F} 6.5 \\ 8-20 \mathrm{~F} 5.1 & 19-5 \mathrm{G} 12.5 \\ 9-20 \mathrm{~F} 5.2 & 20-6 \mathrm{G} 11.4 \\ 10-20 \mathrm{~F} 5.3 & 21-7 \mathrm{G} 9.2\end{array}$

\section{Comments}

Comments are placed at the beginning of the LPF file.

\section{STRESS}

The STRESS menu is used to enter data for all of the stress packages. This menu includes an option for each stress package and the Check option.

\section{Check}

This option checks all the stress data to determine whether data are complete. If not complete, the incomplete data are listed.

\section{River (RIV) Package}

When the internal or external editing mechanism is used to define river reaches with or without parameters, the following fields must be defined for each river reach:

- Layer - the layer number of the cell containing the river reach.

- Row - the row number of the cell containing the river reach.

- Column - the column number of the cell containing the river reach.

- Stage - the head in the river.

- Condfact or Cond-When parameters are used, Condfact is the factor used to calculate riverbed hydraulic conductance from the parameter value. The conductance is the product of Condfact and the parameter value. When parameters are not used, Cond is the riverbed conductance.

- Rbot-the elevation of the bottom of the riverbed.

- IFACE - a flag for use with MODPATH that defines the cell face through which an external source/sink flows. This field is automatically added if MODPATH is activated. IFACE values are:

0 - flow is distributed internally.

1 -flow is applied to the left face.

2 - flow is applied to the right face.

3 - flow is applied to the front face.

4 - flow is applied to the back face.

5 -flow is applied to the bottom face.

6 - flow is applied to the top face.

The "Select Stress Period" list shows all stress periods in the simulation and allows a specific period to be selected.

\section{Parameter Data}

On the "Parameter data" half of the screen, a list shows all the river parameters that have been defined and allows a specific parameter to be selected. The list also shows whether a parameter is active (being used) in the selected stress period.

Five pushbuttons are used to define parameters and control the stress periods in which they are used:

- Deactivate this stress period-deactivates the selected parameter in the selected stress period.

- Activate this stress period - makes the selected parameter active in the selected stress period.

- Modify - allows the selected parameter to be modified.

- New-allows a new parameter to be defined.

- Delete-deletes the selected parameter.

There are two options for specifying the river data for a parameter:

- Separate - allows river data to be located in a file that MFI2005 does not read, and MFI2005 cannot modify that file. Rather, MFI2005 sets up the dataset so that MODFLOW will read the file.

- Package - nonparameter river data are saved in the RIV Package file, and the internal or external editing mechanism is used to modify the data.

When a parameter is first defined using the New pushbutton, the parameter is defined as using the Package option with 0 reaches. 


\section{Nonparameter Data}

Nonparameter river reaches can be defined in addition to the reaches defined by parameters. The choices for defining nonparameter reaches are:

- None-no nonparameter river reaches are being used in the selected stress period.

- Previous - nonparameter river reaches from the previous stress period are reused.

- Package - nonparameter river data are saved in the RIV Package file, and the internal or external editing mechanism is used to modify the data. The fields that must be defined for each reach are the same as for parameters except that Condfact is replaced with the actual riverbed conductance.

- Separate - nonparameter river data are stored in a separate file that MFI2005 does not read.

The Comments pushbutton allows comments to be edited, which are placed at the beginning of the RIV file.

\section{Well (WEL) Package}

When the internal or external editing mechanism is used with or without parameters, the following fields must be defined for each well:

- Layer - the layer number of the cell containing the well.

- Row - the row number of the cell containing the well.

- Column - the column number of the cell containing the well.

- Qfact or Q-When parameters are used, Qfact is the factor used to calculate well recharge rate (negative value indicates pumping) from the parameter value. The recharge rate is the product of Qfact and the parameter value. When parameters are not used, $Q$ is the well recharge rate (negative value indicates pumping)

- IFACE - a flag for use with MODPATH that defines the cell face through which an external source/sink flows. This field automatically is added if MODPATH is activated. IFACE values are:

0 - flow is distributed internally.

1 -flow is applied to the left face.

2-flow is applied to the right face.

3 -flow is applied to the front face.

4 - flow is applied to the back face.

5 -flow is applied to the bottom face.

6-flow is applied to the top face.

The "Select Stress Period" list shows all stress periods in the simulation and allows a specific period to be selected.

\section{Parameter Data}

On the "Parameter data" half of the screen, a list shows all the well parameters that have been defined and allows a specific parameter to be selected. The list also shows whether a parameter is active (being used) in the selected stress period.

Five pushbuttons are used to define parameters and control the stress periods in which they are used:

- Activate this stress period-makes the selected parameter active in the selected stress period.

- Modify—allows the selected parameter to be modified.

- New-allows a new parameter to be defined.

- Delete - deletes the selected parameter.

- Deactivate this stress period-deactivates the selected parameter in the selected stress period.

Two options are available for specifying the well data for a parameter:

- Separate - allows well data to be located in a file that MFI2005 does not read, and MFI2005 cannot modify that file. Rather, MFI2005 sets up the dataset so that MODFLOW will read the file.

- Package - well data are saved in the WEL Package file, and the internal or external editing mechanism is used to modify the data.

A parameter, first defined using the New pushbutton, is defined as using the Package option with 0 wells.

\section{Nonparameter Data}

Nonparameter wells can be defined in addition to the wells defined by parameters. The choices for defining nonparameter wells are:

- None - no nonparameter wells are being used in the selected stress period.

- Previous - nonparameter wells from the previous stress period are reused.

- Package - nonparameter well data are saved in the WEL Package file, and the internal or external editing mechanism is used to modify the data. The fields that must be defined for each well are the same as for parameters except that Qfact is replaced with the actual well recharge (negative value indicates discharge).

- Separate-nonparameter well data are stored in a separate file that MFI2005 does not read.

The Comments pushbutton allows comments, which are placed at the beginning of the WEL file, to be edited. 


\section{Recharge (RCH) Package}

The "Depth Option" list allows the vertical location for applying recharge to be specified:

- Top Layer-recharge is applied to layer 1.

- Specified Layers - recharge is applied to the layer specified in two-dimensional array IRCH. When this option is selected, IRCH appears in the "Array Name" list.

- Automatic Selection - recharge passes vertically through no-flow cells until it reaches a variable-head cell or a constant-head cell. A constant-head cell intercepts the recharge and prevents deeper infiltration.

The "Select Stress Period" list shows all stress periods in the simulation and allows a specific period to be selected.

The "Select Array" list shows the two-dimensional arrays that must be defined without parameters and allows an array to be selected. The RECH array can be defined with or without parameters (but not both). If parameters are defined in the RCH Package, they apply to RECH. Thus, RECH does not appear in the Array Name list if there are any $\mathrm{RCH}$ parameters defined.

\section{Parameter Data}

On the "Parameter data" half of the screen, a list shows all the recharge parameters that have been defined and allows a specific parameter to be selected. The list also shows whether a parameter is active (being used) in the selected stress period.

Five pushbuttons are used to define parameters and control the stress periods in which they are used:

- Activate parameter this stress period - makes the selected parameter active in the selected stress period.

- Modify - allows the selected parameter to be modified.

- New-allows a new parameter to be defined.

- Delete-deletes the selected parameter.

- Deactivate Parameter this stress period-deactivates the selected parameter in the selected stress period.

\section{Nonparameter Data}

Nonparameter arrays are defined the same way as other twodimensional arrays in MFI2005. The choices for defining nonparameter arrays are:

- Previous - nonparameter data from the previous stress period are reused.

- Constant-all cells have the same value.
- Package - nonparameter data are saved in the RCH Package file, and the internal or external editing mechanism is used to modify the data.

- Separate-nonparameter data are stored in a separate file that MFI2005 does not read.

The Comments pushbutton allows comments, which are placed at the beginning of the $\mathrm{RCH}$ file, to be edited.

\section{Drain (DRN) Package}

When the internal or external editing mechanism is used with or without parameters, the following fields must be defined for each drain:

- Layer - the layer number of the cell containing the drain.

- Row - the row number of the cell containing the drain.

- Column - the column number of the cell containing the drain.

- Elevation - the elevation of the drain.

- Condfact or Cond-When parameters are used, Condfact is the factor used to calculate drain conductance from the parameter value. The drain conductance is the product of Condfact and the parameter value. When parameters are not used, Cond is the drain conductance.

- IFACE - a flag for use with MODPATH that defines the cell face through which an external source/sink flows. This field automatically is added if MODPATH is activated. IFACE values are:

0 - flow is distributed internally.

1 -flow is applied to the left face.

2 - flow is applied to the right face.

3 - flow is applied to the front face.

4-flow is applied to the back face.

5-flow is applied to the bottom face.

6- flow is applied to the top face.

The "Select Stress Period" list shows all stress periods in the simulation and allows a specific period to be selected.

\section{Parameter Data}

On the "Parameter data" half of the screen, a list shows all the drain parameters that have been defined and allows a specific parameter to be selected. The list also shows whether a parameter is active (being used) in the selected stress period.

Several pushbuttons are used to define parameters and control the stress periods in which they are used:

- Activate this stress period - makes the selected parameter active in the selected stress period. 
- Modify —allows the selected parameter to be modified.

- New-allows a new parameter to be defined.

- Delete-deletes the selected parameter.

- Deactivate this stress period-deactivates the selected parameter in the selected stress period.

Two options are available for specifying the drain data for a parameter:

- Separate - allows drain data to be located in a file that MFI2005 does not read, and MFI2005 cannot modify that file. Rather, MFI2005 sets up the dataset so that MODFLOW will read the file.

- Package - drain data are saved in the DRN Package file, and the internal or external editing mechanism is used to modify the data.

A parameter, first defined using the New pushbutton, is defined as using the Package option with 0 drains.

\section{Nonparameter Data}

Nonparameter drains can be defined in addition to the drains defined by parameters. The choices for defining nonparameter drains are:

- None-no nonparameter drains are being used in the selected stress period.

- Previous - nonparameter drains from the previous stress period are reused.

- Package - nonparameter drain data are saved in the DRN Package file, and the internal or external editing mechanism is used to modify the data. The fields that must be defined for each drain are the same as for parameters except that Condfact is replaced with the actual drain conductance.

- Separate - nonparameter drain data are stored in a separate file that MFI2005 does not read.

The Comments pushbutton allows comments, which are placed at the beginning of the DRN file, to be edited.

\section{General-Head Boundary (GHB) Package}

When the internal or external editing mechanism is used, the following fields must be defined for each boundary:

- Layer - the layer number of the cell containing the boundary.

- Row - the row number of the cell containing the boundary.

- Column - the column number of the cell containing the boundary.

- Bhead - the head on the boundary.
- Condfact or Cond-When parameters are used, Condfact is the factor used to calculate boundary conductance from the parameter value. The boundary conductance is the product of Condfact and the parameter value. When parameters are not used, Cond is the boundary conductance.

- IFACE - a flag for use with MODPATH that defines the cell face through which an external source/sink flows. This field is automatically added if MODPATH is activated. IFACE values are:

0 - flow is distributed internally.

1 -flow is applied to the left face.

2-flow is applied to the right face.

3 - flow is applied to the front face.

4-flow is applied to the back face.

5 - flow is applied to the bottom face.

6-flow is applied to the top face.

The "Select Stress Period" list shows all stress periods in the simulation and allows a specific period to be selected.

\section{Parameter Data}

On the "Parameter data" half of the screen, a list shows all the GHB parameters that have been defined and allows a specific parameter to be selected. The list also shows if a parameter is active (being used) in the selected stress period.

Several pushbuttons are used to define parameters and control the stress periods in which they are used:

- Activate this stress period - makes the selected parameter active in the selected stress period.

- Modify - allows the selected parameter to be modified.

- New-allows a new parameter to be defined.

- Delete - deletes the selected parameter.

- Deactivate this stress period-deactivates the selected parameter in the selected stress period.

Two options are available for specifying the boundary data for a parameter:

- Separate - allows boundary data to be located in a file that MFI2005 does not read, and MFI2005 cannot modify that file. Rather, MFI2005 sets up the dataset so that MODFLOW will read the file.

- Package - boundary data are saved in the GHB Package file, and the internal or external editing mechanism is used to modify the data.

A parameter, first defined using the New pushbutton, is defined as using the Package option with 0 boundaries. 


\section{Nonparameter Data}

Nonparameter boundaries can be defined in addition to the boundaries defined by parameters. The choices for defining nonparameter boundaries are:

- None - no nonparameter boundaries are being used in the selected stress period.

- Previous - nonparameter boundaries from the previous stress period are reused.

- Package - nonparameter boundary data are saved in the GHB Package file, and the internal or external editing mechanism is used to modify the data. The fields that must be defined for each boundary are the same as for parameters except that Condfact is replaced with the actual boundary conductance.

- Separate - nonparameter boundary data are stored in a separate file that MFI2005 does not read.

The Comments pushbutton allows comments, which are placed at the beginning of the GHB file, to be edited.

\section{Evapotranspiration (EVT) Package}

The "Depth Option" list allows the vertical location for applying evapotranspiration to be specified:

- Top Layer-evapotranspiration is applied to layer 1.

- Specified Layers - evapotranspiration is applied to the layer specified in two-dimensional array IEVT. When this option is selected, IEVT appears in the "Array Name" list.

The "Select Stress Period" list shows all stress periods in the simulation and allows a specific period to be selected.

The "Select Array" list shows the two-dimensional arrays that must be defined without parameters and allows an array to be selected. The EVTR array can be defined with or without parameters (but not both). If parameters are defined in the EVT Package, they apply to EVTR. Thus, EVTR does not show up in the Array Name list if any EVT parameters are defined. The other arrays to be defined are SURF, EXDP, and optionally IEVT.

\section{Parameter Data}

On the "Parameter data" half of the screen, a list shows all the evapotranspiration parameters that have been defined and allows a specific parameter to be selected. The list also shows whether a parameter is active (being used) in the selected stress period.
Nonparameter Data

Five pushbuttons are used to define parameters and control the stress periods in which they are used:

- Activate parameter this stress period-makes the selected parameter active in the selected stress period.

- Modify - allows the selected parameter to be modified.

- New-allows a new parameter to be defined.

- Delete-deletes the selected parameter.

- Deactivate Parameter this stress period-deactivates the selected parameter in the selected stress period.

Nonparameter arrays are defined the same way as other twodimensional arrays in MFI2005. The choices for defining nonparameter arrays are:

- Previous - nonparameter data from the previous stress period are reused.

- Constant—all cells have the same value.

- Package - nonparameter data are saved in the EVT Package file, and the internal or external editing mechanism is used to modify the data.

- Separate - nonparameter data are stored in a separate file that MFI2005 does not read.

The Comments pushbutton allows comments, which are placed at the beginning of the EVT file, to be edited.

\section{SOLVER}

The solver screen allows one of four solvers to be selected for solving the groundwater and sensitivity simultaneous equations:

PCG

MXITER is the maximum number of outer iterations - that is, calls to the solution routine. For a linear problem, MXITER should be 1 , unless more than 50 inner iterations are required, when MXITER could be as large as 10. A larger number (generally less than 100) is required for a nonlinear problem.

ITER1 is the number of inner iterations. For nonlinear problems, ITER1 usually ranges from 10 to 30 ; a value of 30 will be sufficient for most linear problems.

IPRPCG is the printout interval for PCG. If IPRPCG is equal to 0 , the variable is changed to 999 . The maximum head change (positive or negative) and residual change are printed for each iteration of a time step when the time step is an even multiple of IPRPCG. This printout also occurs at the end of each stress period regardless of the value of IPRPCG. 
MUTPCG is a flag that controls printing of convergence information from the solver:

0 - is for printing tables of maximum head change and residual every iteration.

1 -is for printing only the total number of iterations.

2 - is for no printing.

3 - is for printing only if convergence fails.

HCLOSE is the head change criterion for convergence, in units of length. When the maximum absolute value of head change from all nodes during an iteration is less than or equal to HCLOSE, and the criterion for RCLOSE also is satisfied (see below), iteration stops.

RCLOSE is the residual criterion for convergence, in units of cubic length per time. When the maximum absolute value of the residual at all nodes during an iteration is less than or equal to RCLOSE, and the criterion for HCLOSE also is satisfied (see above), iteration stops.

For nonlinear problems, convergence is achieved when the convergence criteria are satisfied for the first inner iteration.

RELAX is the relaxation parameter used with NPCOND $=1$. Usually, RELAX $=1.0$, but for some problems a value of 0.99 , 0.98 , or 0.97 will reduce the number of iterations required for convergence. RELAX is not used if NPCOND is not 1.

DAMP is the damping factor. The damping factor typically is set equal to 1.0 , which indicates no damping. A value less than 1.0 and greater than 0.0 causes damping.

\section{SIP}

MXITER is the maximum number of times through the iteration loop in one time step in an attempt to solve the system of finite-difference equations.

HCLOSE is the head change criterion for convergence. When the maximum absolute value of head change from all nodes during an iteration is less than or equal to HCLOSE, iteration stops.

ACCL is the acceleration variable. The acceleration variable must be greater than 0.0 and is generally equal to 1.0 . If 0.0 is entered, it is changed to 1.0 .

WSEED is the seed for calculating iteration variables. If a value of 0.0 is entered, MFI2005 will specify input data such that SIP will calculate the seed.

IPRSIP is the printout interval for SIP. If IPRSIP is equal to 0 , the variable is changed to 999 . The maximum head change (positive or negative) is printed for each iteration of a time step when the time step is an even multiple of IPRSIP. This printout also occurs at the end of each stress period regardless of the value of IPRSIP.

\section{DE4}

ITMX is the maximum number of iterations for each time step. Specify ITMX $=1$ if iteration is not desired. Ideally, iteration would not be required for direct solution; however, the iteration is necessary if the flow equation is nonlinear (see explanation for IFREQ $=3$ ) or if computer precision limitations result in inaccurate calculations as indicated by a large water budget error ( $>1$ percent for a 10,000-cell grid). For a nonlinear flow equation, each iteration is equally time consuming because $[\mathrm{A}]$ is changed at the start of each iteration and Gaussian elimination is required after each change. Performing Gaussian elimination every iteration is called external iteration. For a linear equation, iteration is appreciably faster because $[\mathrm{A}]$ is changed, at most, once per time step; thus, Gaussian elimination is required, at most, once per time step. Iteration without performing Gaussian elimination every iteration is called internal iteration.

IFREQ is a flag indicating the frequency at which coefficients in [A] change. This affects the efficiency of solution; appreciable work can be avoided if $[\mathrm{A}]$ is known to remain constant all or part of the time.

IFREQ $=1$ indicates that the flow equations are linear and that coefficients of simulated head for all stress terms are constant for all stress periods. To meet the linearity requirement, all model layers must be confined (which is specified in the Block-Centered Flow Package by setting LAYCON equal to 0 for all layers or in the Layer-Property Flow Package by setting LAYTYP equal to 0 for all layers), and formulations must not change based on head (such as seepage from a river changing from head dependent flow to a constant flow when head drops below the bottom of the riverbed). Examples of coefficients of simulated head for stress terms are riverbed conductance, drain conductance, maximum evapotranspiration rate, evapotranspiration extinction depth, and general-head boundary conductance.

IFREQ $=2$ indicates that the flow equations are linear, but coefficients of simulated head for some stress terms may change at the start of each stress period. (See IFREQ $=1$ for information about linear equations.) Examples of coefficients of simulated head for stress terms are riverbed conductance, drain conductance, maximum evapotranspiration rate, evapotranspiration extinction depth, and general-head boundary conductance. For a simulation consisting of only one stress period, IFREQ $=2$ has the same meaning as IFREQ $=1$.

IFREQ $=3$ indicates that a nonlinear flow equation is being solved, which means that some terms in [A] depend on simulated head. Examples of head-dependent terms in $[\mathrm{A}]$ are transmissivity for water-table layers, where transmissivity is based on saturated thickness; flow terms for rivers, drains, and evapotranspiration if they convert between head dependent flow and constant 
flow; and the change in storage coefficient when a cell converts between confined and unconfined. When a nonlinear flow equation is being solved, external iteration (ITMX > 1) normally is required to accurately approximate the nonlinearities. Note that when nonlinearities caused by water-table calculations are part of a simulation, obvious signs are not necessarily produced in the output from a simulation that does not use external iteration to indicate that iteration is needed. In particular, the budget error may be acceptably small without iteration even though appreciable error exists in head because of nonlinearity. To understand this, consider the watertable correction for transmissivity. Each iteration a new transmissivity is calculated based on the previous head. Then the flow equations are solved, and a budget is computed using the new head with the same transmissivities. No appreciable budget discrepancy results because heads are correct for the transmissivity being used at this point; however, the new heads may mean that an appreciable change in transmissivity has occurred. The new transmissivity will not be calculated unless there is another iteration. Therefore, when one or more layers are under water-table conditions, iteration always should be tried. The maximum change in head in each iteration (printed by DE4 when IPRD4 $=1$ and MUTD4 $=0$ ) provides an indication of the effect of all nonlinearities.

HCLOSE is the head change closure criterion. If iterating (ITMX > 1), iteration stops when the absolute value of head change at every node is less than or equal to HCLOSE. HCLOSE is not used if not iterating, but a value always must be specified.

ACCL is a multiplier for the computed head change for each iteration. Normally, this value is 1 . A value greater than 1 may be useful for improving the rate of convergence when using external iteration to solve nonlinear problems $($ IFREQ $=3$ ). ACCL always should be 1 for linear problems. When ITMX $=1, A C C L$ is changed to 1 regardless of the input value; however, a value always must be specified.

IPRD4 is the time-step interval for printing out convergence information when iterating (ITMX $>1$ ). For example, if IPRD4 is 2 , convergence information is printed every other time step. A value always must be specified even if not iterating.

MUTD4 is a flag that indicates the quantity of information that is printed when convergence information is printed for a time step.

MUTD4 $=0$ indicates that the number of iterations in the time step and the maximum head change are printed after each iteration.

MUTD4 = 1 indicates that only the number of iterations in the time step is printed.

MUTD4 $=2$ indicates no information is printed.

MXUP is the maximum number of equations in the upper part of the equations to be solved (Harbaugh, 1995). This value affects the amount of memory used by the DE4 Package. If specified as 0 , the program will calculate MXUP as half the number of cells in the model, which is an upper limit. The actual number of equations in the upper part will be less than half the number of cells whenever no-flow and constant-head cells exist because flow equations are not formulated for these cells. The actual number of equations in the upper part is printed when the DE4 Package runs. The printed value can be used for MXUP in future runs to minimize memory usage.

MXLOW is the maximum number of equations in the lower part of equations to be solved (Harbaugh, 1995). This value affects the amount of memory used by the DE4 Package. If specified as 0 , the program will calculate MXLOW as half the number of cells in the model, which is an upper limit. The actual number of equations in the lower part will be less than half the number of cells whenever no-flow and constant-head cells exist because flow equations are not formulated for these cells. The actual number of equations in the lower part is printed when the DE4 Package runs. The printed value can be used for MXLOW in future runs to minimize memory usage.

MXBW is the maximum band width plus 1 of the $[\mathrm{AL}]$ matrix (Harbaugh, 1995). This value affects the amount of memory used by the DE4 Package. If specified as 0 , the program will calculate MXBW as the product of the two smallest grid dimensions plus 1 , which is an upper limit. The actual band width plus 1 is printed when the DE4 Package runs. The printed value can be used for MXBW in future runs to minimize memory usage.

\section{GMG}

The GMG solver package has both an inner loop and outer loop. The inner loop is set up for the convergence of the linear problem and is controlled by the variables RCLOSE and IITER. A nonlinear problem is controlled through the outer loop and uses variables HCLOSE and MXITER. As a solution of the linear problem is used as an approximate solve for the nonlinear problem, all four variables are needed when solving a nonlinear problem.

RCLOSE is the residual convergence criterion for the inner iteration. The PCG algorithm computes the L2norm of the residual and compares it against RCLOSE. Typically, RCLOSE is set to the same value as HCLOSE (see below). If RCLOSE is set too high, then additional outer iterations may be required due to the linear equation not being solved with sufficient accuracy. On the other hand, a too restrictive setting for RCLOSE for nonlinear problems may force an unnecessarily accurate linear solution. This may be alleviated with the IITER variable or with damping.

IITER is the maximum number of PCG iterations for each linear solution. A value of 100 is typically sufficient. It is frequently useful to specify a smaller number for nonlinear problems in order to prevent an excessive number of inner iterations. 
HCLOSE is the headchange convergence criterion for nonlinear problems. After each linear solve (inner iteration), the maxnorm of the head change is compared against HCLOSE. HCLOSE can be set to a large number for linear problems; HCLOSE is ignored if MXITER $=1$.

MXITER is the maximum number of outeriterations. For linear problems, MXITER can be set to 1. For nonlinear problems, MXITER needs to be larger, but rarely more than 100 .

DAMP is the value of the damping parameter. For linear problems, a value of 1.0 should be used. For nonlinear problems, a value less than 1.0 but greater than 0.0 may be necessary to achieve convergence. A typical value for nonlinear problems is 0.5. Damping also helps control the convergence criterion of the linear solve to alleviate excessive PCG iterations.

IADAMP is a flag that controls adaptive damping. The possible values of IADAMP and their meanings are as follows:

IADAMP $=0$ - the value assigned to DAMP is used as a constant damping parameter.

IADAMP $=1$ - the value of DAMP is used for the first nonlinear iteration. The damping parameter is adaptively varied on the basis of the head change, using Cooley's method as described in Mehl and Hill (2001), for subsequent iterations.

IADAMP $=2$ - the relative reduced residual damping method documented in Mehl and Hill (2001) and modified by Banta (2006) is used.

IOUTGMG is a flag that controls the output of the GMG solver. The possible values of IOUTGMG and their meanings are as follows:

IOUTGMG $=0-$ only the solver inputs are printed.

IOUTGMG $=1$-for each linear solve, the number of PCG iterations, the value of the damping parameter, the L2norm of the residual, and the maxnorm of the headchange and its location (column, row, layer) are printed. At the end of a time/stress period, the total number of GMG calls, PCG iterations, and a running total of PCG iterations for all time/stress periods are printed.

IOUTGMG $=2$ - the convergence history of the PCG iteration is printed, showing the L2norm of the residual and the convergence factor for each iteration.

IOUTGMG $=3$ - the same as IOUTGMG $=1$ except output is sent to the terminal instead of the MODFLOW-2005 LIST output file.

IOUTGMG $=4$ - the same as IOUTGMG $=2$ except output is sent to the terminal instead of the MODFLOW-2005 LIST output file.

IUNITMHC is a flag and a unit number that controls output of maximum head change values. In MFI2005, this field is displayed as a file name. If the name is blank, maximum head change values are not written to an output file. A nonblank name indicates that head change values will be written to that file.
ISM is a flag that controls the type of smoother used in the multigrid preconditioner. The possible values for ISM and their meanings are as follows:

$I S M=0-I L U(0)$ smoothing is implemented in the multigrid preconditioner. This smoothing requires an additional vector on each multigrid level to store the pivots in the ILU factorization.

ISM = 1-Symmetric GaussSeidel (SGS) smoothing is implemented in the multigrid preconditioner. No additional storage is required for this smoother; users may want to use this option if available memory is exceeded or nearly exceeded when using ISM $=0$. Using SGS smoothing is not as robust as ILU smoothing; additional iterations are likely to be required in reducing the residuals. In extreme cases, the solver may fail to converge as the residuals cannot be reduced sufficiently.

ISC is a flag that controls semicoarsening in the multigrid preconditioner. The possible values of ISC and their meanings are given as follows:

ISC $=0$ - the rows, columns, and layers are all coarsened.

ISC $=1$-the rows and columns are coarsened, but the layers are not.

ISC $=2$ - the columns and layers are coarsened, but the rows are not.

ISC $=3$ - the rows and layers are coarsened, but the columns are not.

ISC $=4$ - there is no coarsening.

Typically, the value of ISC should be 0 or 1 . In the case that there are large vertical variations in the hydraulic conductivities, then a value of 1 should be used (see Remark 9 in "CoarseGrid Correction" section in Wilson and Naff, 2004). If no coarsening is implemented (ISC $=4$ ), then the GMG solver is comparable to the PCG2 solver described in Hill (1990) and uses the least amount of memory.

DUP is the maximum damping value that should be applied for an iteration when the solver is not oscillating; DUP is dimensionless.

DLOW is the minimum damping value to be generated by the adaptive-damping procedure; DLOW is dimensionless.

CHGLIMIT is the maximum allowed head change at any cell between outer iterations. DLOW has units of length. The effect of CHGLIMIT is to determine a damping value that will produce a maximum head change equal to CHGLIMIT.

RELAX is a relaxation parameter for the ILU preconditioned conjugate gradient method. The RELAX parameter can be used to improve the spectral condition number of the ILU preconditioned system. The value of RELAX should be approximately one. However, the relaxation parameter can cause the factorization to break down. If this happens, then the GMG solver will report an assembly error and a value smaller than one for RELAX should be tried. This item is read only if ISC $=4$. 


\section{OUTPUT}

The OUTPUT Option (Output Control in MODFLOW) controls the amount of output from MODFLOW. If this option is not used, then default output control is used. Under the default, head and overall budget are written to the listing file (printed) at the end of every stress period. The default printout format for head and drawdown is 10G11.4.

\section{Output File Names and Formats}

"Head Print Format" and "Drawdown Print Format" are codes indicating the format for printing head and drawdown, respectively, in the LIST file (variables IHEDFM and IDDNFM). The codes are as follows:

$\begin{array}{ll}0-10 \mathrm{G} 11.4 & 11-20 \mathrm{~F} 5.4 \\ 1-11 \mathrm{G} 10.3 & 12-10 \mathrm{G} 11.4 \\ 2-9 \mathrm{G} 13.6 & 13-10 \mathrm{~F} 6.0 \\ 3-15 \mathrm{~F} 7.1 & 14-10 \mathrm{~F} 6.1 \\ 4-15 \mathrm{~F} 7.2 & 15-10 \mathrm{~F} 6.2 \\ 5-15 \mathrm{~F} 7.3 & 16-10 \mathrm{~F} 6.3 \\ 6-15 \mathrm{~F} 7.4 & 17-10 \mathrm{~F} 6.4 \\ 7-20 \mathrm{~F} 5.0 & 18-10 \mathrm{~F} 6.5 \\ 8-20 \mathrm{~F} 5.1 & 19-5 \mathrm{G} 12.5 \\ 9-20 \mathrm{~F} 5.2 & 20-6 \mathrm{G} 11.4 \\ 10-20 \mathrm{~F} 5.3 & 21-7 \mathrm{G} 9.2\end{array}$

Further, if the print code is negative, the data are printed in strip format as opposed to wrap format.

"Head Save File," "Drawdown Save File," and "IBOUND Save File" are for specifying the names of files for saving head, drawdown, and IBOUND, respectively. The data only will be saved, however, if the times for saving the data are specified in the other options of Output Control shown below. This screen also includes other options for controlling data formats:

"BINARY" and "ASCII" options - a binary file is written as a nontext file that cannot be read by a typical text editor. These files must be read by special programs that are designed to read MODFLOW's binary (unformatted) data. An ASCII file is a text file that can be read with a typical text editor.

The "Format" field specifies the format for writing an ASCII (text) file. These formats must adhere to Fortran standards, which includes a requirement to enclose the format in parentheses.

"Unlabeled" and "Labeled" options - these are options for an ASCII file. Unlabeled means the data are written without a label specifying the array name and other information such as simulation time. Labeled means there is a one-line label.

Cell-by-Cell Budget Files

The "Compact" and "Original" radio buttons determine the budget file format. Original is the original format used for MODFLOW. Compact is an optional format in MODFLOW-2005 that stores data using less disk space.
"All Data to One File" and "Specify File for Each Package" radio buttons control which files are used for saving budget data. "All Data to One File" indicates that all budget terms are written into the same file, and that file is specified in the "Name" field. "Specify File for Each Package" means that for each package that can save budget data, a file name for saving the data can be entered into the table. If the name is blank, no budget data are written for that package. If the file name is "PRINT" for WEL, RIV, GHB, DRN, BCF, or LPF, then budget data are written to the listing file.

\section{Times for Printing Head}

This option allows the times for printing head in the LIST file to be specified. The options are "None," "Simulation End," "Every Stress Period," "All Time Steps," and "List." The first four of these options require no additional input. The "List" option requires the user to specify a list of stress periods and time steps at which head should be printed.

\section{Times for Saving Head}

This option allows the times for saving head to be specified. The options are the same as for printing head. Note that head will not be saved unless a file name is specified using the "Output File Names" option above.

\section{Times for Printing Drawdown}

This option allows the times for printing drawdown in the LIST file to be specified. The options are the same as for printing head.

\section{Times for Saving Drawdown}

This option allows the times for saving drawdown to be specified. The options are the same as for printing head. Note that drawdown will not be saved unless a file name is specified using the "Output File Names" option above.

\section{Times for Printing Budget}

This option allows the times for printing the overall budget in the LIST file to be specified. The options are the same as for printing head, except that "None" and "Simulation End" cannot be selected because the budget must be printed at least at the end of every stress period.

\section{Times for Saving Flow (CBC) Data}

This option allows the times for saving budget data to be specified. The options are the same as for printing head. Note that budget data will not be saved unless a file name (or names) is specified using the "Output File Names" option above.

\section{Times for Saving IBOUND}

This option allows the times for saving IBOUND to be specified. The options are the same as for printing head. Note that IBOUND will not be saved unless a file name is specified using the "Output File Names" option above. 


\section{Comments}

Comments are placed at the beginning of the Output Control file.

\section{MODPATH}

Prepare data for running MODPATH.

\section{Check}

This option checks the MODPATH data to determine whether data are complete. If not complete, the incomplete data are listed.

Misc.

Maximum number of particles-Specify 0 to use the default incorporated in MODPATH.

Maximum size of composite budget file-This value is used only for transient simulations. Specify 0 to use the default maximum file size. If the maximum is exceeded, MODPATH prompts the user to determine if it is OK to exceed that size.

Output style-MODPATH can save data in various formats, and this option allows the format to be chosen.

Modpath-Plot Water Table-Modpath-Plot can draw the water table using various line types, and this option allows the line type to be chosen.

Time assigned to the beginning of the MODFLOW simulation-MODFLOW always assumes that the time is 0.0 at the beginning of a simulation, but MODPATH allows a different time to be specified.

Time range for data in budget file- To save disk space in transient simulations, MODFLOW requires only that budget data generated by MODFLOW be saved for the duration of time for which particles are tracked.

\section{IBOUND}

MODPATH includes an IBOUND variable that is similar to MODFLOW's IBOUND. One value is read for every model cell. In addition to indicating variable head $(>0)$, no flow $(=0)$, and constant head $(<0)$, specific positive IBOUND values are used in MODPATH to indicate plotting colors and areas for stopping particles. The following editing options allow the MODPATH IBOUND values to be defined for each layer:

- Constant - all elements of the array have the same value.

- Package - data are saved in the MODPATH MAIN data file, and the internal or external editing mechanism is used to modify the data.

- Separate - data are stored in a separate file that MFI2005 does not read.
When MODPATH is first activated in MFI2005, MODFLOW IBOUND values are used as initial values for MODPATH IBOUND if the Constant or Package options are used for MODFLOW.

\section{Porosity}

Darcy velocity is divided by porosity to obtain actual velocity. The following editing options allow the porosity values to be defined for each layer:

- Constant - all elements of the array have the same value.

- Package - data are saved in the MODPATH MAIN data file, and the internal or external editing mechanism is used to modify the data.

- Separate - data are stored in a separate file that MFI2005 does not read.

\section{GUA Method}

Specifies how the Grid Unit Array (GUA) is used when plotting.

- None-GUA is not used.

- ZONES - The GUA is an Integer array, and the value at each cell determines the color of the cell when the GUA is plotted.

- Nonuniform RANGES-The GUA is a Real array. A table of arbitrary numeric ranges is defined, and each range is assigned a number. The color plotted is determined from the range number to which the GUA value corresponds.

- Uniform RANGES-The GUA is a Real array. A table of numeric ranges with a uniform interval is defined, and each range is assigned a number. The color plotted is determined from the range number to which the GUA value corresponds.

\section{Grid Unit Array}

The following editing options allow the GUA to be defined for each layer:

- Constant-all elements of the array have the same value.

- Package - data are saved in the MODPATH MAIN data file, and the internal or external editing mechanism is used to modify the data.

- Separate - data are stored in a separate file that MFI2005 does not read. 


\section{Observations}

\section{Sngl. Time Head}

This option allows head observations for single times to be specified. One variable, a table, and two special pushbuttons are displayed.

Variables:

TOMULTH is the time-offset multiplier for head observations. The product of TOMULTH and TOFFSET must produce a time value in the units used for all other model input data. The same variable appears in the Multi-Time Head screen.

Output File is the name of a file for saving the simulated equivalents.

HOBDRY is the simulated equivalent that is written if the cell at the observation location is dry.

Table:

Each row in the table can contain one observation; the fields in the table are described below. Rows in which the "Obs. Name" field is blank are ignored.

Obs. Name is a string of 1 to 12 nonblank characters used to identify the observation.

Layer is the layer in which the observation is located. If an observation is for a single layer, the layer can be edited directly in the table. If an observation is multilayer, the layer field is disabled, and the Edit Multilayer pushbutton must be used to specify the layers.

Row is the row in which the observation is located.

Column is the column in which the observation is located.

IREFSP is the stress period to which the observation time (TOFFSET) is referenced. The reference point is the beginning of stress period IREFSP.

TOFFSET is the time from the beginning of stress period IREFSP to the time of the observation.

HOBS is the observed head.

ROFF is the row offset used to locate the observation in the finite-difference cell. For an observation in row i, 0.0 is the center of the cell, -0.5 is the face between rows $\mathrm{i}-1$ and $\mathrm{i}$, and +0.5 is the face between rows $\mathrm{i}$ and $\mathrm{i}+1$.

COFF is the column offset used to locate the observation in the finite-difference cell. For an observation in column $\mathrm{j}, 0.0$ is the center of the cell, -0.5 is the face between columns $j-1$ and $j$, and +0.5 is the face between columns $\mathrm{j}$ and $\mathrm{j}+1$.

Statistic is the value from which the observation weight is calculated when UCODE_2005 is used.
StatFlag is a flag identifying the type of statistic used to calculate the observation weight when UCODE_2005 is used:

$$
\begin{aligned}
& \text { Variance-scaled variance } \\
& \text { Stnd. Dev.-scaled standard deviation } \\
& \text { Coef. of Var.-scaled coefficient of variation } \\
& \text { WT-weight }
\end{aligned}
$$$$
\text { SQRWT-square root of the weight }
$$

PlotSymbol is an integer that UCODE_2005 writes to output files intended for graphical analysis to allow control of the symbols used to plot data.

Pushbuttons:

Edit Multilayer-allows multiple model layers to be assigned to observations. Additional dialogs allow an observation to be selected and the layers to which the observation corresponds to be specified. For each layer, the proportion of the simulated head that is used to calculate the simulated multilayer head also is specified.

Comments - allows comments to be included in the head observations file.

\section{Multi-Time Head}

This option allows head observations for multiple times at the same location to be specified. One variable, a table, and three special pushbuttons are displayed.

Variables:

TOMULTH is the time-offset multiplier for head observations. The product of TOMULTH and TOFFSET must produce a time value in the units used for all other model input data. The same variable appears in the Sngl. Time Head screen.

Output File is the name of a file for saving the simulated equivalents.

HOBDRY is the simulated equivalent that is written if the cell at the observation location is dry.

Table:

Each row in the table shows a summary of information for one location with multi-time observations.

Pushbuttons:

Comments - allows comments to be included in the head observations file.

New Multi-Time Observation-allows a new location at which multi-time observations can be specified. This starts another dialog that allows the observation to be defined. The additional dialog is the same as the dialog for editing multi-time observations.

Edit Multi-Time Observation - allows the selected observation to be modified using a separate dialog. 
Edit Multi-Time Observation Dialog_Five individual fields, radio buttons, and two tables:

Header Name is a string of 1 to 12 nonblank characters used to identify the location, which is displayed in the Multi-Time head Dialog.

Row is the row in which the observation is located.

Column is the column in which the observation is located.

Row Offset (variable ROFF) is used to locate the observation in the finite-difference cell. For an observation in row i, 0.0 is the center of the cell, -0.5 is the face between rows $i-1$ and $i$, and +0.5 is the face between rows $i$ and $i+1$.

Column Offset (variable COFF) is used to locate the observation in the finite-difference cell. For an observation in column $\mathrm{j}, 0.0$ is the center of the cell, -0.5 is the face between columns $j-1$ and $j$, and +0.5 is the face between columns $\mathrm{j}$ and $\mathrm{j}+1$.

Radio Buttons:

Use the buttons to specify whether the objective function is based on head or drawdown.

First Table:

Layer is the layer in which the observation applies. If there are multiple rows in which Layer $>0$, the observation is multi-layer.

Proportion is the proportion of the simulated head that is used to calculate the simulated multilayer head. If there is only one row in which Layer $>0$, proportion is not used.

Second Table:

Name is a string of 1 to 12 nonblank characters used to identify the observation.

IREFSP is the stress period to which the observation time (TOFFSET) is referenced. The reference point is the beginning of stress period IREFSP.

TOFFSET is the time from the beginning of stress period IREFSP to the time of the observation.

HOBS is the observed head.

Statistic is the value from which the observation weight is calculated when UCODE_2005 is used.

StatFlag is a flag identifying the type of statistic used to calculate the observation weight when UCODE_2005 is used:

Variance - scaled variance

Stnd. Dev.- scaled standard deviation

Coef. of Var.- - scaled coefficient of variation

WT-weight

SQRWT—-square root of the weight
PlotSymbol is an integer that UCODE 2005 writes to output files intended for graphical analysis to allow control of the symbols used to plot data.

\section{GHB Observations}

Time offset multiplier (variable TOMULTGB) - The product of TOMULTGB and TOFFSET (in the observation time dia$\log$ ) must produce a time value in the units used for all other model input data.

Output File is the name of a file for saving the simulated equivalents.

The list shows all of the observation groups that have been defined.

"DELETE Cell group" causes the selected group to be deleted.

"ADD Cell group" causes a new observation group to be created with 0 times and locations.

"EDIT Observation Times" allows observed values to be specified for multiple times. A separate screen with the following fields is activated:

OBSNAM is a string of 1 to 12 nonblank characters used to identify the observation.

IREFSP is the stress period to which the observation time (TOFFSET) is referenced. The reference point is the beginning of stress period IREFSP.

TOFFSET is the time from the beginning of stress period IREFSP to the time of the observation.

HOBS is the observed inflow to the groundwater system from the boundary (negative indicates outflow).

Statistic is the value from which the observation weight is calculated when UCODE_2005 is used.

StatFlag is a flag identifying the type of statistic used to calculate the observation weight when UCODE_2005 is used:

\section{Variance - scaled variance}

Stnd. Dev. - scaled standard deviation

Coef. of Var.- -scaled coefficient of variation

WT-weight

SQRWT—square root of the weight

PlotSymbol is an integer that UCODE_2005 writes to output files intended for graphical analysis to allow control of the symbols used to plot data.

"EDIT Cell Locations" allows the cells that define the location of the observation to be specified. A separate screen with the following fields is activated:

Layer is the layer number of a boundary cell that is in the cell group. 
Row is the row number of a boundary cell that is in the cell group.

Column is the column number of a boundary cell that is in the cell group.

FACTOR is the portion of the simulated flow for this cell that is included in the total simulated flow for this cell group.

"Force Factor $=1.0$ for all cells" is a check box that causes FACTOR to be set to 1.0 for all cells in the cell group when MODLFOW runs regardless of the values for FACTOR in the table.

\section{DRN Observations}

Time offset multiplier (variable TOMULTDR) - The product of TOMULTDR and TOFFSET (in the observation time dia$\log$ ) must produce a time value in the units used for all other model input data.

Output File is the name of a file for saving the simulated equivalents.

The list shows all of the observation groups that have been defined.

"DELETE Cell group" causes the selected group to be deleted.

"ADD Cell group" causes a new observation group to be created with 0 times and locations.

"EDIT Observation Times" allows observed values to be specified for multiple times. A separate screen with the following fields is activated:

OBSNAM is a string of 1 to 12 nonblank characters used to identify the observation.

IREFSP is the stress period to which the observation time (TOFFSET) is referenced. The reference point is the beginning of stress period IREFSP.

TOFFSET is the time from the beginning of stress period IREFSP to the time of the observation.

HOBS is the observed inflow to the groundwater system from the boundary (negative indicates outflow). The value must be negative for the Drain Package.

Statistic is the value from which the observation weight is calculated when UCODE_2005 is used.

StatFlag is a flag identifying the type of statistic used to calculate the observation weight when UCODE_2005 is used:

Variance - scaled variance

Stnd. Dev. - scaled standard deviation

Coef. of Var.-scaled coefficient of variation

WT—weight

SQRWT—square root of the weight
PlotSymbol is an integer that UCODE 2005 writes to output files intended for graphical analysis to allow control of the symbols used to plot data.

"EDIT Cell Locations" allows the cells that define the location of the observation to be specified. A separate screen with the following fields is activated:

Layer is the layer number of a drain cell that is in the cell group.

Row is the row number of a drain cell that is in the cell group.

Column is the column number of a drain cell that is in the cell group.

FACTOR is the portion of the simulated flow for this cell that is included in the total simulated flow for this cell group.

"Force Factor $=1.0$ for all cells" is a check box that causes FACTOR to be set to 1.0 for all cells in the cell group when MODLFOW runs regardless of the values for FACTOR in the table.

\section{RIV Observations}

Time offset multiplier (variable TOMULTRV) - The product of TOMULTRV and TOFFSET (in the observation time dia$\log$ ) must produce a time value in the units used for all other model input data.

Output File is the name of a file for saving the simulated equivalents.

The list shows all of the observation groups that have been defined.

"DELETE Cell group" causes the selected group to be deleted.

"ADD Cell group" causes a new observation group to be created with 0 times and locations.

"EDIT Observation Times" allows observed values to be specified for multiple times. A separate screen with the following fields is activated:

OBSNAM is a string of 1 to 12 nonblank characters used to identify the observation.

IREFSP is the stress period to which the observation time (TOFFSET) is referenced. The reference point is the beginning of stress period IREFSP.

TOFFSET is the time from the beginning of stress period IREFSP to the time of the observation.

HOBS is the observed inflow to the groundwater system from the river (negative indicates outflow).

Statistic is the value from which the observation weight is calculated when UCODE_2005 is used. 
StatFlag is a flag identifying the type of statistic used to calculate the observation weight when UCODE_2005 is used:

Variance-scaled variance

Stnd. Dev. - scaled standard deviation

Coef. of Var.-scaled coefficient of variation

WT-weight

SQRWT — square root of the weight

PlotSymbol is an integer that UCODE_2005 writes to output files intended for graphical analysis to allow control of the symbols used to plot data.

"EDIT Cell Locations" allows the cells that define the location of the observation to be specified. A separate screen with the following fields is activated:

Layer is the layer number of a river cell that is in the cell group.

Row is the row number of a river cell that is in the cell group.

Column is the column number of a river cell that is in the cell group.

FACTOR is the portion of the simulated flow for this cell that is included in the total simulated flow for this cell group.

"Force Factor $=1.0$ for all cells" is a check box that causes FACTOR to be set to 1.0 for all cells in the cell group when MODLFOW runs regardless of the values for FACTOR in the table.

\section{Constant-head Obs.}

Time offset multiplier (variable TOMULTCH)—The product of TOMULTCH and TOFFSET (in the observation time dia$\log$ ) must produce a time value in the units used for all other model input.

Output File is the name of a file for saving the simulated equivalents.

The list shows all of the observation groups that have been defined.

"DELETE Cell group" causes the selected group to be deleted.

"ADD Cell group" causes a new observation group to be created with 0 times and locations.

"EDIT Observation Times" allows observed values to be specified for multiple times. A separate screen with the following fields is activated:

OBSNAM is a string of 1 to 12 nonblank characters used to identify the observation.
IREFSP is the stress period to which the observation time (TOFFSET) is referenced. The reference point is the beginning of stress period IREFSP.

TOFFSET is the time from the beginning of stress period IREFSP to the time of the observation.

HOBS is the observed inflow to the groundwater system from the boundary (negative indicates outflow).

Statistic is the value from which the observation weight is calculated when UCODE_2005 is used.

StatFlag is a flag identifying the type of statistic used to calculate the observation weight when UCODE_2005 is used:

$$
\begin{aligned}
& \text { Variance-scaled variance } \\
& \text { Stnd. Dev.—-scaled standard deviation } \\
& \text { Coef. of Var.-scaled coefficient of variation } \\
& \text { WT_-weight } \\
& \text { SQRWT_-square root of the weight }
\end{aligned}
$$

PlotSymbol is an integer that UCODE_2005 writes to output files intended for graphical analysis to allow control of the symbols used to plot data.

"EDIT Cell Locations" allows the cells that define the location of the observation to be specified. A separate screen with the following fields is activated:

Layer is the layer number of a boundary cell that is in the cell group.

Row is the row number of a boundary cell that is in the cell group.

Column is the column number of a boundary cell that is in the cell group.

FACTOR is the portion of the simulated flow for this cell that is included in the total simulated flow for this cell group.

"Force Factor $=1.0$ for all cells" is a check box that causes FACTOR to be set to 1.0 for all cells in the cell group when MODLFOW runs regardless of the values for FACTOR in the table.

\section{Parameter Values}

The Parameter Values screen allows values for all parameters to be changed from a single screen. A pushbutton and a table are displayed.

Pushbutton:

"Select Parameters to be Included in PVAL File" allows any currently defined parameter to be included in the Parameter Value File. 
Table - The table contains the following fields for each parameter that will be included in the Parameter Value File:

PARNAM is the name of a previously defined parameter. This field cannot be modified.

Parval is the new value of the parameter, which overrides the value specified when the parameter was defined.

GWF File Value is the value of the parameter that was specified when the parameter was defined. This value cannot be modified in the Parameter Values screen. The GWF File Value is not used as long as the parameter is contained in the Parameter Value file. GWF File Value is shown in this screen for information purposes.

\section{The following fields are included in the Parameter Value screen for use by UCODE_2005; these values have no impact on a MODFLOW $\bar{W}$ simulation:}

StartValue is the starting parameter value when performing parameter estimation.

LowerValue is the smallest reasonable parameter value. This value does not restrict the estimated value when parameter estimation is used. This value is printed in the model output to facilitate comparison with the estimated value.

UpperValue is the largest reasonable parameter value. This value does not restrict the estimated value when parameter estimation is used. This value is printed in the model output to facilitate comparison with the estimated value.

ScalePval is an alternate value for the parameter. If the absolute value of the parameter is less than ScalePval, ScalePval replaces the parameter in the scaling of onepercent, dimensionless, and composite scaled sensitivities. Good choices are the minimum (in absolute value) reasonable value of the parameter or a value two or three orders of magnitude smaller than the parameter value.

Transform is a check box field that indicates whether the parameter is log-transformed for parameter estimation.

Adjustable is a check box field that indicates whether UCODE can compute sensitivities and change the value if parameter estimation is being performed.

Constrain is a check box that indicates whether UCODE constrains the parameter values when performing parameter estimation.

LowerConstraint is the lower limit of a constrained parameter.

UpperConstraint the upper limit of a constrained parameter.

\section{UCODE}

This screen allows the UCODE_2005 program (Poeter and others, 2005) to be controlled. Many variables and a table of prior information are displayed.

Variables:

Verbose is a flag that controls the amount of output in the main UCODE output file:

No extraneous output

Warnings

Warnings, notes

Warnings, notes, echo selected input

Warnings, notes, echo all input

Warnings, notes, echo all input plus

miscellaneous information

A set of buttons indicates the mode of UCODE_2005only one can be selected:

Sensitivities-Forward-Calculate sensitivities using forward-difference perturbation.

Sensitivities-Central-Calculate sensitivities using central-difference perturbation.

Optimize-Estimate parameters.

Linearity-Execute the test-model-linearity mode.

Linearity $A d v=$ conf - Execute the advanced-modellinearity mode using parameter values from the data exchange file with_bladvconf as the file name extension.

Linearity Adv=pred-Execute the advanced-modellinearity mode using parameter values from the data exchange file with_bladvpred as the file name extension.

SOSSurface - Calculate sum-of-squared weighted residuals objective function values for the parameters designated as 'Adjustable.'

SOSIncrement is the number of values to be considered between and including the LowerConstraint and UpperConstraint when SOSSurface is being used.

StartRes specifies that residuals for the starting parameter values should be printed.

IntermedRes specifies that residuals should be printed after each parameter-estimation iteration.

FinalRes specifies that residuals for final parameter values should be printed.

StartSens controls printing of starting parameter sensitivities:

css - Composite scaled sensitivities 
dss_-Dimensionless and composite scaled sensitivities

onepercentss-One-percent scaled sensitivities and composite scaled sensitivities

allss - Composite, dimensionless, and one-percent scaled sensitivities

unscaled-Unscaled and composite scaled sensitivities

all-All

none

IntermedSens controls printing of parameter sensitivities after each parameter-estimation iteration:

css-Composite scaled sensitivities

dss-Dimensionless and composite scaled sensitivities

onepercentss - One-percent scaled sensitivities and composite scaled sensitivities

allss - Composite, dimensionless, and one-percent scaled sensitivities

unscaled-Unscaled and composite scaled sensitivities

all-All

none

FinalSens controls printing of final parameter sensitivities:

css-Composite scaled sensitivities

dss-Dimensionless and composite scaled sensitivities

onepercentss - One-percent scaled sensitivities and composite scaled sensitivities

allss-Composite, dimensionless, and one-percent scaled sensitivities

unscaled-Unscaled and composite scaled sensitivities

all-All

none

EigenValues specifies that eigenvalues should be computed and printed.

CreateInitFiles specifies that data-exchange files will be generated with file name extensions _init, _init._mv, _init._su, and, if prior information is specified,_init._supri.

ModelMassUnits defines the mass units used in the model.
TolPar is the parameter-estimation closure criterion, as a fractional change in parameter values. TOL commonly equals 0.01 . Larger values often are used during preliminary calibration efforts; values as small as 0.001 can be used.

TolSOSC is the second convergence criterion. If SOSC $\neq 0.0$, parameter estimation stops if the fractional change in the sum-of-squared objective function is less than TolSOSC. SOSC usually equals 0.0. Typical nonzero values of SOSC are 0.01 and 0.05 . If TolSOSC $=0.0$, SOSC convergence is not used.

MaxIter is the maximum number of parameterestimation iterations allowed before stopping.

MaxChange is the maximum fractional amount parameter values are allowed to change in one iteration. MaxChange commonly equals 2.0 or less if parameter values are unstable during parameter-estimation iterations.

MaxChangeRealm specifies the transformation space to which MaxChange applies, which can be Native or Regression. For Regression, MaxChange applies to logtransformed values.

TrustRegion specifies if the trust-region modification of Gauss-Newton regression is used with the step size determined by the double-dogleg strategy.

MaxStep specifies the maximum allowable step size for the double-dogleg strategy. The default is a function of the parameter values and is printed in the main UCODE_2005 output file. Specify -1 to use the default.

ConsecMax is the maximum number of times that MaxStep can be used consecutively for the double-dogleg strategy before execution stops.

MqrtDirection is the angle (in degrees) between the downgradient direction on the sum-of-squared-residuals surface and the parameter update vector above which the Marquardt parameter is applied. If this angle approaches $90^{\circ}$, regression is unlikely to make progress. Default $=85.4^{\circ}$.

MqrtFactor is a constant used to compute the Marquardt parameter. Default $=1.5$.

MqrtIncrement is a constant used to compute the Marquardt parameter. Default $=0.001$.

OmitInsensitive specifies that composite scaled sensitivities (CSS) are used to omit parameters from the regressions and reinclude them.

MinimumSensRatio is used to determine if parameters are omitted when OmitInsensitive is used. Default $=0.005$. 
ReincludeSensRatio is used to determine if parameters are reincluded when OmitInsensitive is used. Default $=0.02$.

Table of prior information-Each row defines one prior information equation:

PriorName is a user-supplied name for a priorinformation equation.

PriorInfoValue is the prior estimate for the priorinformation equation.

Statistic is the value from which the weight for the priorinformation equation is calculated.

StatFlag a flag identifying the type of statistic used by UCODE_2005 to compute the weight:

Variance - scaled variance

Stnd. Dev. - scaled standard deviation

Coef. of Var.- - scaled coefficient of variation

WT-weight

SQRWT—-square root of the weight

PlotSymbol is an integer that UCODE_2005 writes to output files intended for graphical analysis to allow control of the symbols used when plotting data related to prior information.

Equation defines how to calculate PriorName from model information.

\section{External Editing Mechanism}

Arrays or lists that are defined using the package method will be included in the primary data file for the package or option to which the data belong. For example, if the array of recharge rates is defined using the package method, the array will be included in the RCH file. When using MFI2005, package data can be edited using an internal screen or an external program such as a text editor or a spreadsheet program. The external editing mechanism is activated when the "Send for External Editing" pushbutton is selected. The external editing mechanism consists of the following steps:

1. When the mechanism is activated, MFI2005 writes the current values of the array or list into a file named mfiss.csv. This file is a comma-delimited text file.

2. MFI2005 displays a message box telling the user to edit miniss.csv.
3. The user should not immediately respond to the message box. Rather, the user should activate the external program and edit_mfiss.csv as desired. A typical text editor, such as Notepad, can be used as the editor.

4. When data editing has been completed, the user must save the modified data back into_mfiss.csv using a commadelimited structure. The fields must be separated by a comma with optional additional spaces, and each field must have a nonblank value. However, the width of each field does not have to be the same.

5. The user must then close mfiss.csv in the external program. The external program need not be terminated provided_mfiss.csv can be closed without terminating the editing program.

6. The user should respond with OK to the MFI2005 message box.

7. MFI2005 will then read the modified values that were placed back into_mfiss.csv by the external program.

8. MFI2005 continues processing user commands.

\section{Instructions for using Excel as the external editing program:}

Reading_mfiss.csv_-Use the Open option in Excel, and select the file_mfiss.csv in the directory in which MFI2005 is running.

Saving data back into_mfiss.csv-The quickest way to complete steps 4-5 above is to use the Close option in Excel without separately specifying the Save option:

1. Choose the Close option.

2. If the data have been modified, Excel always asks if the data should be saved. Respond with "Yes."

3. The "Save As" screen then will display the file name (which should still should be mfiss.csv). Respond with OK.

4. Respond with "Yes" to the prompt to replace the existing file.

5. Finally, Excel will inform the user that a text file cannot save all information in a spreadsheet and will ask if the user wants to leave the file in this format. Respond with "Yes" to keep the comma-delimited text format. 


\section{Prepared by:}

USGS Enterprise Publishing Network

Raleigh Publishing Service Center

3916 Sunset Ridge Road

Raleigh, NC 27607

For additional information regarding this publication, contact:

U.S. Geological Survey

Office of Ground Water

411 National Center

Reston, VA 20192

(703) 648-5001

Or visit the USGS Groundwater Software Web site at: http://water.usgs.gov/software/lists/groundwater/ 

